\title{
Long-term outcome of stereotactic aspiration, endoscopic evacuation, and open craniotomy for the treatment of spontaneous basal ganglia hemorrhage: a propensity score study of 703 cases
}

\author{
Yong Du ${ }^{1 \#}$, Yuan Gao ${ }^{2 \#}$, Hai-Xiao Liu ${ }^{1 \#}$, Long-Long Zheng ${ }^{1}$, Zhi-Jun Tan ${ }^{3}$, Hao Guo ${ }^{1}$, Xun Wu ${ }^{1}$, \\ Wen-Xing Cui ${ }^{1}$, Chen Yang ${ }^{1}$, Ying-Wu Shi ${ }^{1}$, Gao-Yang Zhou ${ }^{1}$, Fei-Fei Sun ${ }^{1}$, Rui-Xi Fan ${ }^{1}$, Tian Feng ${ }^{1}$, \\ Ping Wang ${ }^{1}$, Lei Wang ${ }^{1}$, Wei Guo ${ }^{1}$, Yan $\mathrm{Qu}^{1}$ \\ ${ }^{1}$ Department of Neurosurgery, Tangdu Hospital, the Fourth Military Medical University, Xi'an, China; ${ }^{2}$ School of Aerospace Medicine, The Fourth \\ Military Medical University, Xi'an, China; ${ }^{3}$ Department of Health Statistics, The Fourth Military Medical University, Xi'an, China \\ Contributions: (I) Conception and design: Y Du, Y Gao, HX Liu; (II) Administrative support: Y Qu, W Guo; (III) Provision of study materials \\ or patients: LL Zheng, H Gao, X Wu, WX Cui, C Yang, YW Shi; (IV) Collection and assembly of data: GY Zhou, FF Sun, RX Fan, T Feng, P \\ Wang, L Wang; (V) Data analysis and interpretation: Y Du, Y Gao, HX Liu, ZJ Tan; (VI) Manuscript writing: All authors; (VII) Final approval of \\ manuscript: All authors. \\ \#Those authors contributed equally to this work. \\ Correspondence to: Yan Qu, MD, PhD; Wei Guo, MD, PhD. Department of Neurosurgery, Tangdu Hospital, the Fourth Military Medical University, \\ Xi’an 710038, China. Email: yanqu0123@fmmu.edu.cn; 18729985168@163.com.
}

Background: To compare the long-term therapeutic effects of stereotactic aspiration (SA), endoscopic evacuation (EE), and open craniotomy (OC) in the surgical treatment of spontaneous basal ganglia hemorrhage and explore the appropriate clinical indications for each technique.

Methods: Multiple-treatment inverse probability of treatment weighting (IPTW)-adjusted logistic regression analysis was performed to evaluate the therapeutic effects of these techniques. The primary and secondary outcomes were 6-month modified Rankin Scale (mRS) and mortality rates, respectively.

Results: A total of 703 patients were ultimately enrolled. For the entire cohort, the 6-month mortality rate was significantly higher (OR 2.396, 95\% CI: 1.865-3.080), and the 6-month functional outcome was significantly worse (OR 1.359, 95\% CI: 1.091-1.692) for SA than that of EE. The 6-month mortality rate for OC was significantly higher (OR 1.395, 95\% CI: 1.059-1.837) than that of EE. Further subgroup analysis was stratified by initial hematoma volume and Glasgow Coma Scale (GCS) score. The mortality rate for SA was significantly higher for patients with hematoma volume of 20-40 mL (OR 6.226, 95\% CI: 3.848-10.075), 40-80 mL (OR 2.121, 95\% CI: 1.492-3.016), and $\geq 80 \mathrm{~mL}$ (OR 5.544, 95\% CI: 3.315-9.269) than in the same subgroups of EE. The functional outcomes for SA were significantly worse than that of EE for hematoma volume subgroups of $40-80 \mathrm{~mL}$ (OR 1.424, 95\% CI: 1.039-1.951) and $\geq 80 \mathrm{~mL}$ (OR 4.224, 95\% CI: 1.655-10.776). The mortality rate for SA was significantly higher than that of EE for the GCS score subgroups of 6-8 (OR 2.082, 95\% CI: 1.410-3.076) and 3-5 (OR 2.985, 95\% CI: 1.904-4.678). The mortality rate for OC was significantly higher for the GCS score of 3-5 subgroup (OR 1.718, 95\% CI: 1.115-2.648), and a tendency for a higher mortality rate of 6-8 subgroup (OR 1.442, 95\% CI: 0.965-2.156) than that of EE.

Conclusions: EE can decrease the 6-month mortality rate and improve the 6-month functional outcomes of spontaneous basal ganglia hemorrhage in patients with a hematoma volume $\geq 40 \mathrm{~mL}$. EE can decrease the 6-month mortality rate of spontaneous basal ganglia hemorrhage in patients with a GCS score of 3-8.

Keywords: Basal ganglia hemorrhage; minimally invasive surgery; endoscopic evacuation; open craniotomy (OC); propensity score 
Submitted Apr 02, 2021. Accepted for publication Jul 08, 2021.

doi: 10.21037/atm-21-1612

View this article at: https://dx.doi.org/10.21037/atm-21-1612

\section{Introduction}

Spontaneous intracerebral hemorrhage (ICH) is the most common and fatal hemorrhagic stroke with the highest rate of morbidity and mortality among all types (1). The overall mortality rate of ICH within 30 days after onset is $>30 \%$ (2), and only $25 \%$ of patients can live independently at 6 months (3). It imposes huge health and economic burden on families and societies (4).

Conservative treatment and surgical evacuation are the main treatments for spontaneous ICH (5). The mass effect of the hematoma and the toxic effects of blood degradation products on the surrounding tissues can be alleviated by surgical evacuation of the hematoma when necessary.

Currently, the main surgical treatments of supratentorial spontaneous ICH are stereotactic aspiration (SA), endoscopic evacuation (EE), and open craniotomy (OC). It is believed that patients with particular characteristics [e.g., Glasgow Coma Scale (GCS) score and hematoma volume] might benefit from different surgical treatments (6). However, the patients enrolled in previous studies have been highly heterogeneous or chosen for a certain characteristic (7-9). Furthermore, subgroup analysis of patients with ICH according to hematoma volume and GCS scores remains insufficient, and the ideal candidate for each surgery has not been determined $(10,11)$.

To that end, we conducted a retrospective study with inverse probability of treatment weighting (IPTW)-adjusted analysis to compare the 6-month function outcomes and 6-month mortality rates of patients with basal ganglia hemorrhage who underwent surgical treatment. Further subgroup analysis took the GCS score and the hematoma volume as stratified factors to explore the long-term efficacy of the three surgical methods and the appropriate clinical indications for each surgical technique.

We present the following article in accordance with the STROBE reporting checklist (available at https://dx.doi. org/10.21037/atm-21-1612).

\section{Methods}

\section{Study design and population}

The purpose of this study was to compare long-term outcomes, including the 6-month modified Rankin Scale (mRS) score and 6-month mortality rate in the patients with spontaneous basal ganglia hemorrhage who received one of the following surgical techniques: SA, EE, and OC. The medical records of all patients diagnosed as ICH at Tangdu Hospital between January 1, 2015, and December 30, 2019, were reviewed retrospectively. This study was approved by the Biological and Medical Ethics Committee of Tangdu Hospital (No. TDLL-2014115), and the requirement for informed consent was waived. The study was conducted in accordance with the Declaration of Helsinki (as revised in 2013). Patients were selected according to the following inclusion and exclusion criteria.

\section{Inclusion criteria}

(I) Age >20 years old;

(II) Diagnosed with spontaneous basal ganglia hemorrhage by computed tomography (CT);

(III) Hematoma volume $\geq 20 \mathrm{~mL}$;

(IV) Underwent SA, EE, or OC;

(V) Hospitalized within $24 \mathrm{~h}$ after onset.

\section{Exclusion criteria}

(I) Hemorrhage caused by secondary factors (arteriovenous malformation, intracranial aneurysm, coagulopathy, intracranial tumor, or trauma);

(II) Multiple ICHs;

(III) Known to have advanced dementia or disability before $\mathrm{ICH}$;

(IV) Any severe illnesses that would interfere with assessment of prognosis, including cardiac, hepatic, renal, gastroenterological, respiratory, endocrinological, immunological, or hematological disease;

(V) Using antiplatelet or anticoagulant drugs or had concurrent coagulation disorders;

(VI) Brain stem affected by hematoma;

(VII) Refusal or loss of follow-up.

\section{Treatments}

All patients received standard medical treatments in a dedicated stroke unit according to the recommendations of the American Heart Association/American Stroke 
Association (AHA/ASA). CT scans, routine blood tests, coagulation function tests, and biochemical examinations were performed immediately after admission. In addition, the results of neurological physical examination and medical history of the patients were recorded immediately on admission, and vital signs were monitored.

\section{Surgical procedures}

An experienced surgical team performed all operations. According to the patient's general condition, lifesaving treatment would be conducted if necessary, including decompressive craniotomy, lumbar puncture, external lumbar drainage, and tracheotomy. The principle of minimal invasiveness was followed in all operations. The surgical procedure was based on the methods described in previous studies (12-14). In addition, a CT scan was performed for each patient $24 \mathrm{~h}$ after surgery to evaluate the effect of hematoma evacuation.

In the clinical treatment process, surgeons emergent selected the surgical technique according to the volume, location, and progression of the hematoma, the patient's general condition, the patient's family's wishes, and the surgeons' experience. For patients with larger hematoma volume or lower GCS scores, the probability of intracranial hypertension or cerebral herniation is higher during the operation, surgeons might more quickly manage the emergent intraoperative intracranial hypertension or cerebral herniation when choosing OC.

For the SA procedure, a stereotactic head frame or scale was used to locate the hematoma position according to the preoperative CT scan, avoiding important blood vessels and functional areas. A burr hole was drilled $(\approx 1$ $\mathrm{cm}$ in diameter) under general anesthesia. After the dura was incised, a soft catheter was inserted along the long axis of the hematoma to a predetermined depth. The clot was pumped with a syringe until the first resistance. A postoperative CT scan was performed to determine the location of the catheter and the stability of the residual hematoma. Urokinase was then injected directly into the hematoma cavity through the catheter at a dose of 10,000 30,000 units $/ 2 \mathrm{~mL}$, followed by $3 \mathrm{~mL}$ rinsing every $12 \mathrm{~h}$ for 2-4 days. According to the CT scan, the extubation time was determined according to the drainage volume, the color of the drainage fluid, and the condition. The drainage tube was generally retained for 2-7 days.

For the EE procedure, a transtemporal or transfrontal approach was selected following the principle of the route that provided the shortest distance between the cortical surface and the hematoma according to the preoperative CT scan. The skin was incised under general anesthesia, and a bone flap around $3 \mathrm{~cm}$ in diameter was drilled. The dura mater was incised in a cruciate fashion, the puncturing cannula was placed in a predetermined position of the hematoma cavity, and then the cannula's core was removed. The clot was aspirated with a syringe, and the position of the transparent sheath adjusted as required. Rinsing and aspiration were repeated to evacuate the hematoma as much as possible, without damaging the blood vessel wall, surrounding brain tissue, or causing new bleeding, to achieve the purpose of effectively reducing intracranial pressure.

For the OC procedure, the neurosurgeon performed a traditional craniotomy under microscopic guidance, and the transcortical or transtemporal approach was adopted to evacuate the hematoma. In addition, decompressive craniotomy and tracheotomy were conducted if necessary.

\section{Data collection and outcomes evaluation}

Basic information (sex, age, diagnosis, etc.) was provided by the patient information management department of the hospital. In addition, the following information was collected from the inpatient medical record system: (I) disease history (diabetes, smoking, hypertension) and history of craniocerebral disease including cerebral hemorrhage, cerebral infarction, and traumatic brain injury; (II) treatment information (surgical method, decompressive craniectomy, lumbar puncture, external lumbar drainage, tracheotomy, rehabilitation treatment); and (III) preoperative status of the patient (hematoma volume, GCS score, herniation, interval between onset and operation). No ethnic-based differences were shown in the present study.

The hematoma volume was calculated using the formula $A^{*} B^{*} C / 2$, where $A$ is the greatest diameter on the largest hemorrhage slice, $\mathrm{B}$ is the maximal diameter perpendicular to $\mathrm{A}$, and $\mathrm{C}$ is the vertical hematoma depth.

The patients were followed up by telephone at 1, 3, and 6 months to record their prognostic information after surgery. The primary outcome was the 6-month functional outcome evaluated by the mRS score: $0-3$ was regarded as good; 4-6 was regarded as poor. The secondary outcome was the 6-month mortality rate. Further subgroup analyses of functional outcome and mortality were stratified by hematoma volume ( $\geq 20-40 \mathrm{~mL}, \geq 40-80 \mathrm{~mL}, \geq 80 \mathrm{~mL}$ ) and 
GCS score [3-5, 6-8, 9-14].

\section{Statistical analysis}

All variables were converted to categorical variables and presented as frequency (percentages). One-way ANOVA and chi-square tests were used for univariate comparison. Firstly, the chi-square test was adopted to identify the primary and secondary outcomes and surgical type factors. To minimize the confounding effect from other risk factors, a propensity score weighting for multiple treatments was performed when the risk effect of surgical type on the primary and secondary outcomes was estimated. Covariates, including sex, age, diabetes, smoking, hypertension, hypertension grade, history of the craniocerebral disease, decompressive craniectomy, lumbar puncture, external lumbar drainage, tracheotomy, rehabilitation treatment information, hematoma volume, GCS score, herniation, and the interval between onset and operation were used for calculation of the propensity score by Toolkit for Weighting and Analysis of Nonequivalent Groups (twang package in $\mathrm{R})$. The inverse probability of weights of the propensity scores were calculated by a generalized boosted model with 5000 regression trees to obtain the optimal balance of the propensity score among groups $(b<0.20$ was indicative of good balance of model, Figures S1-S7) (15). Then, hypothesis tests ( $t$-test or chi-square test) and standardized mean differences were performed to evaluate the effect of weights on the magnitude of each confounding factors in the IPTW model (16). Finally, the inverse probability of treatment weighted odds ratio (IPTW-OR) was derived for the surgical groups, and all the enrolled cases were included in the final effect analysis. In the subgroup analysis, a similar IPTW-adjusted logistic regression analysis was adopted. However, the adjusted covariates in each subgroup were not consistent because the primary and secondary outcome's risk factors in different subgroups were not totally the same, and the grouping factor of the subgroup analysis was not included in the IPTW model (Figures S8-S14). In addition, due to the limitation of the number of patients who underwent OC enrolled in GCS score 9-14 and hematoma volume of $20-40 \mathrm{~mL}$ subgroups, and the imbalance of those subgroups, the therapeutic effect between OC and EE in those subgroups were not been evaluated.

Analysis was performed using SAS 9.4 (SAS Institute, Inc., Cary, NC, USA) and R 3.2.4 (R Foundation for Statistical Computing, Vienna, Austria).

\section{Results}

\section{Patient numbers}

1786 supratentorial spontaneous ICH patients were hospitalized from January 1, 2015, to December 30, 2019. Of them, 1012 meet the inclusion criteria, 241 were excluded for not meeting the selection criteria, and 68 were excluded for incomplete essential information. Finally, a total of 703 patients were enrolled in this study (Figure 1).

\section{Basic characteristics}

In the entire cohort, 343 patients were enrolled in the SA group, 212 in the EE group, and 148 in the OC group; 446 were male, and 261 patients were $>60$ years old. The numbers of patients with a history of diagnosed diabetes, smoking, craniocerebral disease, and diagnosed hypertension were 48 (6.83\%), 408 (58.04\%), 89 (12.66\%), and $575(81.79 \%)$, respectively. In addition, preoperative cerebral herniation occurred in $128(18.21 \%)$ patients (Table 1). The mean age, GCS score, the interval between onset and operation, and hematoma volume of the entire cohort were $56.74 \pm 11.07$ years, $7.88 \pm 3.04$ points, $15.81 \pm 14.16 \mathrm{~h}$, and $59.32 \pm 29.88 \mathrm{~mL}$, respectively.

Intergroup equilibrium analysis was conducted to identify the potential confounding factors. GCS score $(\mathrm{P}<0.0001)$, smoking $(\mathrm{P}<0.0001)$, herniation $(\mathrm{P}<0.0001)$, the interval between onset and operation $(\mathrm{P}<0.0001)$, hematoma volume $(\mathrm{P}<0.0001)$, rehabilitation treatment $(\mathrm{P}<0.0001)$, decompressive craniectomy $(\mathrm{P}<0.0001)$, tracheotomy $(\mathrm{P}<0.0001)$, and lumbar puncture $(\mathrm{P}<0.0001)$ were unbalanced variables in the entire cohort. Detailed information regarding the basic characteristics of the entire cohort is presented in Table 1, and the information of each subgroup is presented in Tables $\mathrm{S} 1$ and $\mathrm{S} 2$.

The characteristics that needed adjustments were incorporated into the multivariate IPTW-adjusted logistic regression analysis to minimize the influence of confounding factors. Detailed information regarding the adjusted covariates and balance in each subgroup is presented in Figures S8-S14.

\section{Outcome assessment of entire cohort}

In the entire cohort, the 6-month poor functional outcome (mRS score $>3$ ) rate was $55.76 \%$ (392/703 patients), and the 6 -month mortality rate was $32.57 \%$ (229/703 patients). The 


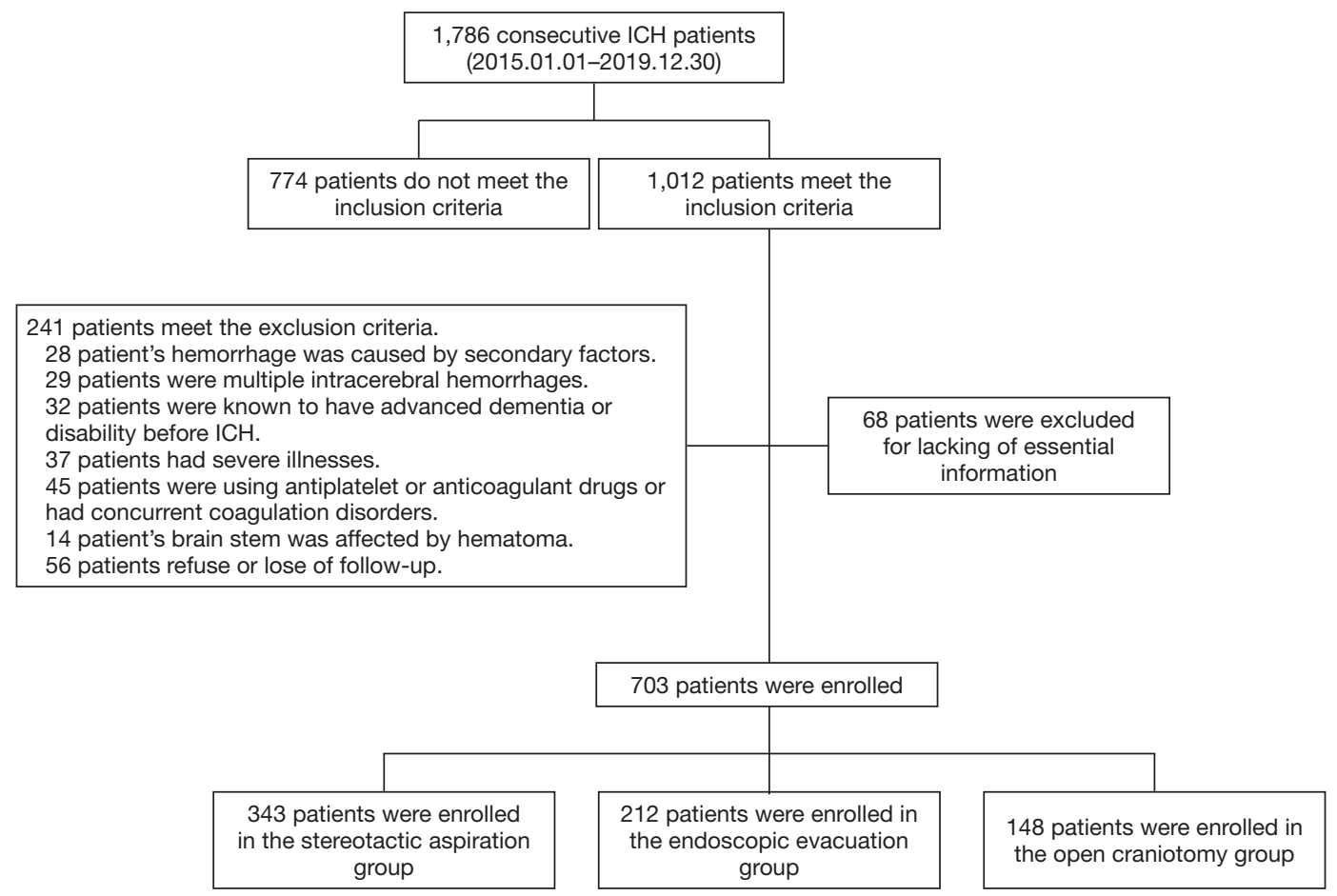

Figure 1 Flowchart of patient selection in this study.

numbers of patients who had a poor functional outcome in the SA, EE, and OC groups were 181 (52.77\%), 94 (44.34\%), and 117 (79.05\%), respectively. The mortality rate in the SA, EE, and OC groups was 124 (36.15\%), 34 (16.04\%), and $71(47.97 \%)$, respectively. The 6-month $\mathrm{mRS}$ score of the entire cohort is presented in Figure S15A.

Multivariate logistic regression analysis showed that the 6-month functional outcome was significantly worse [odds ratio (OR) 1.746, 95\% CI: 1.135-2.686], and the 6-month mortality rate was significantly higher (OR 3.045, 95\% CI: 1.831-5.062) in the SA group than in the EE group. On the other hand, there was no significant difference in the 6-month functional outcome (OR 0.768, 95\% CI: 0.358 1.648 ) and mortality (OR 1.422, 95\% CI: 0.781-2.588) observed between the OC and EE groups (Table 2).

After IPTW adjustment, the results showed that the 6-month functional outcome was significantly worse (OR 1.359, 95\% CI: 1.091-1.692) and the 6-month mortality rate significantly higher (OR 2.396, 95\% CI: $1.865-3.080)$ in the SA group than in the EE group. The 6-month mortality rate was significantly higher (OR 1.395, 95\% CI: $1.059-1.837)$ in the OC group than in the EE group. However, no significant difference in the 6-month functional outcome was observed between the EE and OC groups (OR 0.956, 95\% CI: 0.765-1.194; Table 2).

\section{Outcome assessment by hematoma volume}

Subgroup analysis was performed using the hematoma volume on admission. The distributions of 6-month mRS scores in each hematoma volume subgroup are presented in Figure S15B-S15D.

For the patients with a $20-40 \mathrm{~mL}$ hematoma volume, the 6-month mortality rate in the SA group was significantly higher (OR 6.226, 95\% CI: 3.848-10.075) than in the EE group. In addition, the patients treated by SA also showed a tendency of worse functional outcomes (OR 1.453, 95\% CI: $0.952-2.217)$.

For the patients with a hematoma volume of 40 $80 \mathrm{~mL}$, the 6-month functional outcome was significantly worse (OR 1.424, 95\% CI: 1.039-1.951), and the 6-month mortality was significantly higher (OR $2.121,95 \% \mathrm{CI}$ : $1.492-3.016)$ in the SA group in the EE group. On the other hand, there was no significant difference between the $\mathrm{EE}$ and $\mathrm{OC}$ groups in the 6-month functional outcome (OR 0.894 , 95\% CI: $0.629-1.269$ ) or mortality rate (OR 1.039 , 
Table 1 The inter-group balance tests of possible confounding factors among different surgical techniques

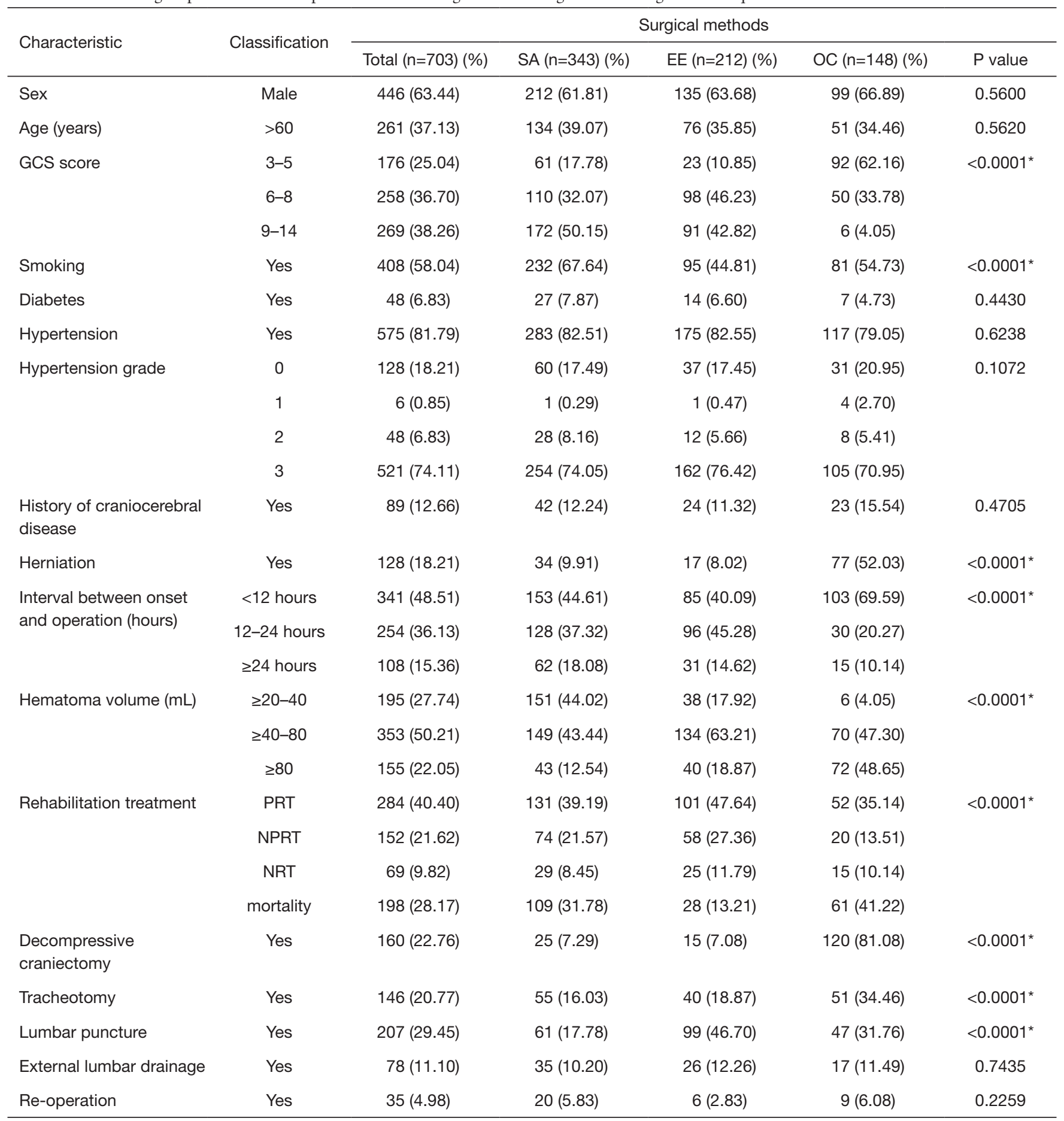

*, the difference has statistical significance. SA, stereotactic aspiration; EE, endoscopic evacuation; OC, open craniotomy; GCS, glasgow coma scale; PRT, professional rehabilitation treatment; NPRT, un-professional rehabilitation treatment; NRT, no rehabilitation treatment. 


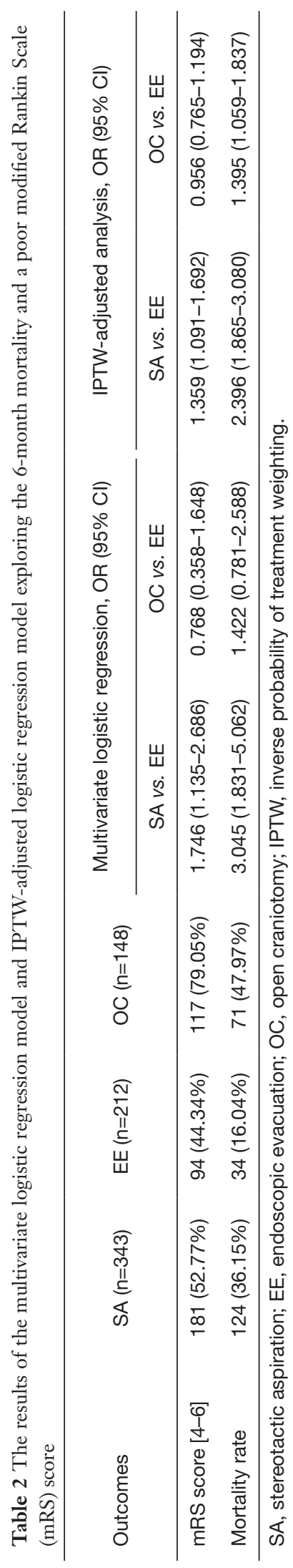

95\% CI: 0.693-1.559).

For the patients with a hematoma volume $\geq 80 \mathrm{~mL}$, the 6 -month functional outcome was significantly worse (OR 4.224, 95\% CI: 1.655-10.776) and the 6-month mortality significantly higher (OR 5.544, 95\% CI: 3.315-9.269) in the SA group than in the EE group. Compared with the EE group, the 6-month mortality in the OC group was significantly higher (OR 2.009, 95\% CI: 1.251-3.226). However, no significant difference existed between OC and EE groups in the 6-month functional outcome (OR 0.967, 95\% CI: 0.506-1.850; Figure 2).

\section{Outcome assessment by GCS score}

Subgroup analysis was performed according to the GCS score on admission. The distribution of the 6-month $\mathrm{mRS}$ scores in each GCS score subgroup is presented in Figure S15E-S15G.

For patients with GCS scores 3-5, the 6-month mortality rate in the SA group was significantly higher (OR 2.985, 95\% CI: 1.904-4.678) than that in the EE group. However, no significant difference was found in 6-month functional outcomes between the SA and EE groups (OR 1.868, 95\% CI: 0.885-3.940). Compared with the EE group, the 6-month mortality rate of the OC group was significantly higher (OR 1.718, 95\% CI: 1.115-2.648). However, no significant difference was observed in 6-month functional outcomes between the OC and EE groups (OR 0.980, 95\% CI: $0.515-1.866)$.

For the patients with GCS score 6-8, the 6-month mortality of the SA group was significantly higher (OR 2.082, 95\% CI: 1.410-3.076) than that of the EE group. However, there was no significant difference in 6-month functional outcomes between the SA and EE groups (OR 1.206, 95\% CI: 0.834-1.744). Compared with the EE group, the OC group tended for a higher mortality rate (OR 1.442, 95\% CI: 0.965-2.156), although no significant difference was observed. In addition, there was no significant difference in 6-month functional outcomes (OR 0.802, 95\% CI: 0.554 1.163) between the OC and EE groups.

For the patients with GCS score 9-14, no significant difference was found in the 6-month functional outcomes and mortality rates between the SA and EE groups. However, a tendency for the worse 6-month functional outcome (OR 1.323, 95\% CI: 0.918-1.907) and higher mortality rate (OR 1.523, 95\% CI: 0.929-2.496) were observed in the SA group, compared with the EE group (Figure 2). 
A

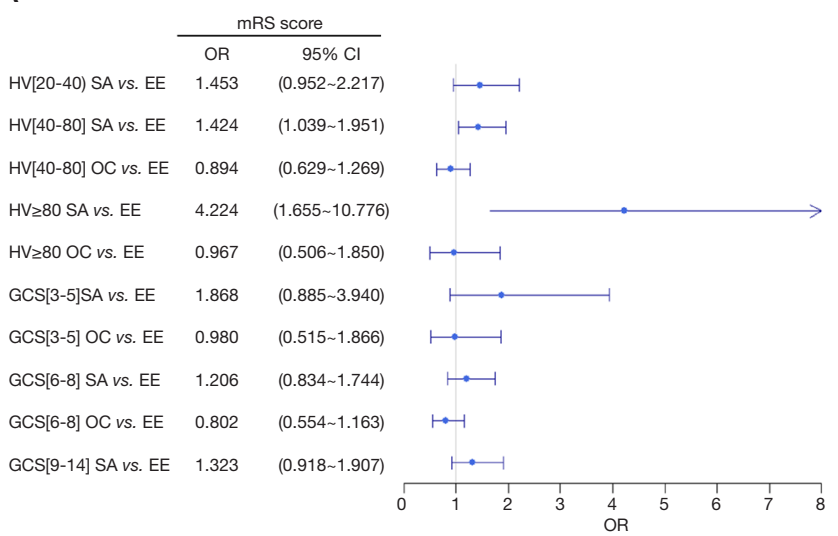

B

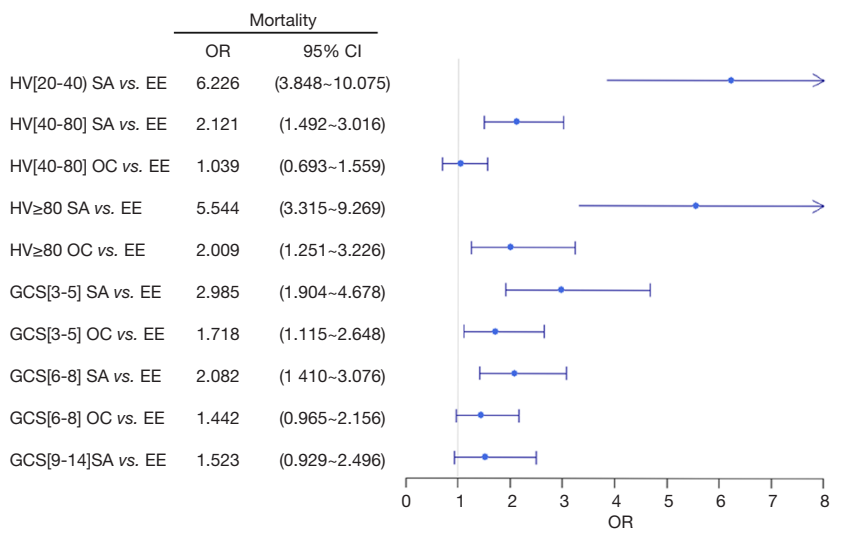

Figure 2 Forest plot showing the IPTW-OR associated with a poor mRS score and death in different subgroups; the reference is endoscopic evacuation. inverse probability of treatment weighted odds ratio; mRS, modified Rankin Scale; SA, stereotactic aspiration; EE, endoscopic evacuation; OC, open craniotomy.

\section{Discussion}

This study aimed to evaluate the long-term therapeutic effect of patients with spontaneous basal ganglia hemorrhage who underwent SA or EE or OC surgical treatment. As indicated by our data, $\mathrm{EE}$ decreased the 6-month mortality rate and improved the 6-month functional outcomes of spontaneous basal ganglia hemorrhage in patients with a hematoma volume $\geq 40 \mathrm{~mL}$. In addition, $\mathrm{EE}$ also decreased the 6-month mortality of spontaneous basal ganglia hemorrhage in patients with GCS scores 3-8.

A previous study found that the GCS score and hematoma volume were the significant independent predictors of death in ICH patients (17). In this present study, subgroup analysis was conducted to explore the appropriate surgical treatment for patients with various GCS scores and hematoma volumes on admission. According to the initial GCS score [3-5, 6-8, 9-14] and hematoma volume ( $\geq 20-40 \mathrm{~mL}, \geq 40-80 \mathrm{~mL}, \geq 80 \mathrm{~mL})$, patients were further divided into six subgroup.

The results of specific subgroup analysis showed that in the GCS score 3-5 subgroup, the 6-month mortality rates of the SA and OC groups were significantly higher than that of the EE group. In the GCS score 6-8 subgroup, the 6-month mortality rate in the SA group was significantly higher, and there was a tendency of higher mortality in the OC group compared with the EE group. This suggested that, for patients with a GCS score of 3-8, EE may be the most appropriate surgical method among the three different techniques. In the hematoma volume of $40-80 \mathrm{~mL}$ and $\geq 80 \mathrm{~mL}$ subgroups, the 6-month functional outcomes were significantly worse, and the mortality rate was significantly higher in the SA group than in the EE group. In the hematoma volume $\geq 80 \mathrm{~mL}$ subgroup, the mortality rate was significantly higher in the OC group than in the EE group. This suggested that, with a hematoma volume of $40-80 \mathrm{~mL}$, EE or OC may be more appropriate, but for hematoma volume $\geq 80 \mathrm{~mL}$, EE may be the most appropriate surgical method. Those results may help to determine the appropriate indications for each surgical method.

One thing worth noting is that compared with SA, EE could significantly improve functional outcomes in the patients with a hematoma volume $\geq 40 \mathrm{~mL}$, which is different from our previous study (18). In this study, the proportion of $\mathrm{mRS}=3$ in the SA, EE, and OC groups of the entire cohort was $20.4 \%, 32.5 \%$, and $18.2 \%$, respectively. The proportion of $\mathrm{mRS}=3$ in the EE group was much higher than in the other two surgical groups. When $\mathrm{mRS}=3$ was attributed to good functional outcomes, we found that EE could decrease the 6-month mortality rate and improve functional outcomes compared with the SA groups. However, there was no significant difference in functional outcomes between the EE and SA in the subgroup analysis of GCS score.

Brain injury caused by ICH includes primary and secondary injury. Primary brain injury is mainly caused by direct traumatic damage to the regional neurons during the hematoma formation (19). The GCS score mainly shows brain injury severity (20). The lower the GCS score on admission, the more serious the direct traumatic damage to the regional neurons. Our results showed no 
significant difference among the three surgical methods in the subgroup analysis according to the GCS score on admission. One possible explanation for this result is that surgery cannot alleviate the direct neuronal damage caused by primary brain injury.

On the other hand, secondary brain injury is mainly caused by a cascade of events initiated by the hematoma and its degradation products, resulting in insufficient cerebral blood supply, increased intracranial pressure, and cerebral hernia (21). Clinical evidence shows that the risk of death from secondary brain injury is higher than for primary brain injury, leading to the deterioration of neurological function or even death $(22,23)$. In the subgroup analysis of hematoma volume, the results showed a significant difference in functional outcome between SA and EE in the patients with a hematoma volume $\geq 40 \mathrm{~mL}$, but there was no significant difference between $\mathrm{OC}$ and $\mathrm{EE}$. The reason may be that both OC and EE have a high evacuation rate, thus effectively preventing secondary brain injury. In contrast, SA usually takes several days to remove the hematoma (24), leading to residual hematoma and perihematomal edema that continue to damage the brain tissue.

Recently, the results of the MISTIE III (Minimally Invasive Surgery Plus Rt-PA for ICH Evacuation) study showed that the MISTIE procedure was safe. However, the percentage of patients with a favorable functional outcome according to mRS (0-3) at 12 months in the surgical treatment group (44.2\%) was not significantly larger than that in the conservative treatment group (41.7\%) (25). However, secondary results suggest that surgical treatment can slightly reduce the mortality rate (25). Furthermore, our results showed that the therapeutic effect of EE was better than that of SA, which is probably because EE has the same minimally invasive characteristics as SA and has the advantages of high hematoma clearance rate, short operation time, and direct visualization $(26,27)$. Interestingly, subgroup analysis in the MISTIE III trial showed that patients with postoperative residual hematoma $\leq 15 \mathrm{~mL}$ had better neurological outcomes than patients with residual hematoma $>15 \mathrm{~mL}$ (28). This suggests that residual hematoma and perihematomal edema are related to the prognosis of neurological function. Further studies are needed to explore the relationship between residual hematoma volume, perihematomal edema, and prognosis with the different surgical methods.

Nevertheless, this study had several limitations that need to be acknowledged. First, it was retrospective, so the possibility of selection bias cannot be ruled out. IPTW- adjusted logistic regression analysis was conducted to minimize the influence of confounding factors between surgical groups on the effect analysis. Second, due to the limitation of the number of patients enrolled in this study, only 6 patients who underwent OC were enrolled in the GCS score 9-14 and hematoma volume of 20-40 mL subgroups. Thus, we did not evaluate the therapeutic effect between OC and EE. Third, only patients with spontaneous basal ganglia hemorrhage were enrolled because the basal ganglia location is the most frequently affected area. Physicians at our center considered that basal ganglia hemorrhage was one subgroup among the supratentorial locations that would potentially benefit most from surgical evacuation. Fourth, this was a single-center study and may have limited generalizability. Therefore, the effectiveness of those surgical techniques needs to be demonstrated in multicenter randomized controlled trials.

\section{Conclusions}

EE decreased the 6-month mortality rate in our cohort and improved the 6-month functional outcomes of spontaneous basal ganglia hemorrhage in patients with a hematoma volume $\geq 40 \mathrm{~mL}$. EE can also decrease the 6-month mortality rate of spontaneous basal ganglia hemorrhage in patients with GCS scores 3-8. Further subgroup analyses explored the therapeutic effects of three surgical techniques and provided a reference for clinicians when choosing the most appropriate method for the patient. Those preliminary results warrant a further large, prospective, randomized study.

\section{Acknowledgments}

Funding: This study was supported by the National Natural Science Foundation of China (81630027) and the China Postdoctoral Science Foundation (2018M633751).

\section{Footnote}

Reporting Checklist: The authors have completed the STROBE reporting checklist. Available at https://dx.doi. org/10.21037/atm-21-1612

Data Sharing Statement: Available at https://dx.doi. org/10.21037/atm-21-1612

Peer Review File: Available at https://dx.doi.org/10.21037/ atm-21-1612 
Conflicts of Interest: All authors have completed the ICMJE uniform disclosure form (available at https:// dx.doi.org/10.21037/atm-21-1612). The authors report that this study was supported by the National Natural Science Foundation of China (81630027) and the China Postdoctoral Science Foundation (2018M633751).

Ethical Statement: The authors are accountable for all aspects of the work in ensuring that questions related to the accuracy or integrity of any part of the work are appropriately investigated and resolved. The study was conducted in accordance with the Declaration of Helsinki (as revised in 2013). The protocol for this retrospective study was approved by the Biological and Medical Ethics Committee of Tangdu Hospital (No. TDLL-2014115) and individual consent for this retrospective analysis was waived.

Open Access Statement: This is an Open Access article distributed in accordance with the Creative Commons Attribution-NonCommercial-NoDerivs 4.0 International License (CC BY-NC-ND 4.0), which permits the noncommercial replication and distribution of the article with the strict proviso that no changes or edits are made and the original work is properly cited (including links to both the formal publication through the relevant DOI and the license). See: https://creativecommons.org/licenses/by-nc-nd/4.0/.

\section{References}

1. Krishnamurthi RV, Feigin VL, Forouzanfar MH, et al. Global and regional burden of first-ever ischaemic and haemorrhagic stroke during 1990-2010: findings from the Global Burden of Disease Study 2010. Lancet Glob Health 2013;1:e259-81.

2. An SJ, Kim TJ, Yoon BW. Epidemiology, Risk Factors, and Clinical Features of Intracerebral Hemorrhage: An Update. J Stroke 2017;19:3-10.

3. Poon MT, Fonville AF, Al-Shahi Salman R. Long-term prognosis after intracerebral haemorrhage: systematic review and meta-analysis. J Neurol Neurosurg Psychiatry 2014;85:660-7.

4. Wang W, Jiang B, Sun H, et al. Prevalence, Incidence, and Mortality of Stroke in China: Results from a Nationwide Population-Based Survey of 480687 Adults. Circulation 2017;135:759-71.

5. Goyal N, Tsivgoulis G, Malhotra K, et al. Minimally invasive endoscopic hematoma evacuation vs. best medical management for spontaneous basal-ganglia intracerebral hemorrhage. J Neurointerv Surg 2019;11:579-83.

6. Mitchell P, Gregson B. Surgery for ICH after MISTIE III. Br J Neurosurg 2019;33:117-8.

7. Feng Y, He J, Liu B, et al. Endoscope-Assisted Keyhole Technique for Hypertensive Cerebral Hemorrhage in Elderly Patients: A Randomized Controlled Study in 184 Patients. Turk Neurosurg 2016;26:84-9.

8. Zhang $\mathrm{X}$, Zhou S, Zhang Q, et al. Stereotactic aspiration for hypertensive intracerebral haemorrhage in a Chinese population: a retrospective cohort study. Stroke Vasc Neurol 2019;4:14-21.

9. Liu H, Wu X, Tan Z, et al. Long-Term Effect of Endoscopic Evacuation for Large Basal Ganglia Hemorrhage With GCS Scores $\leqq 8$. Front Neurol 2020;11:848.

10. Hemphill JC 3rd, Greenberg SM, Anderson CS, et al. Guidelines for the Management of Spontaneous Intracerebral Hemorrhage: A Guideline for Healthcare Professionals From the American Heart Association/ American Stroke Association. Stroke 2015;46:2032-60.

11. Steiner T, Al-Shahi Salman R, Beer R, et al. European Stroke Organisation (ESO) guidelines for the management of spontaneous intracerebral hemorrhage. Int J Stroke 2014;9:840-55.

12. Xu X, Chen X, Li F, et al. Effectiveness of endoscopic surgery for supratentorial hypertensive intracerebral hemorrhage: a comparison with craniotomy. J Neurosurg 2018;128:553-9.

13. Sun GC, Chen XL, Hou YZ, et al. Image-guided endoscopic surgery for spontaneous supratentorial intracerebral hematoma. J Neurosurg 2017;127:537-42.

14. Li Z, Li Y, Xu F, et al. Minimal invasive puncture and drainage versus endoscopic surgery for spontaneous intracerebral hemorrhage in basal ganglia. Neuropsychiatr Dis Treat 2017;13:213-9.

15. McCaffrey DF, Griffin BA, Almirall D, et al. A tutorial on propensity score estimation for multiple treatments using generalized boosted models. Stat Med 2013;32:3388-414.

16. Austin PC, Stuart EA. Moving towards best practice when using inverse probability of treatment weighting (IPTW) using the propensity score to estimate causal treatment effects in observational studies. Stat Med 2015;34:3661-79.

17. Sloan MA, Sila CA, Mahaffey KW, et al. Prediction of 30day mortality among patients with thrombolysis-related intracranial hemorrhage. Circulation 1998;98:1376-82.

18. Guo W, Liu H, Tan Z, et al. Comparison of endoscopic evacuation, stereotactic aspiration, and craniotomy for treatment of basal ganglia hemorrhage. J Neurointerv Surg 
2020;12:55-61.

19. Xi G, Keep RF, Hoff JT. Mechanisms of brain injury after intracerebral haemorrhage. Lancet Neurol 2006;5:53-63.

20. Brennan PM, Murray GD, Teasdale GM. Simplifying the use of prognostic information in traumatic brain injury. Part 1: The GCS-Pupils score: an extended index of clinical severity. J Neurosurg 2018;128:1612-20.

21. Qureshi AI, Mendelow AD, Hanley DF. Intracerebral haemorrhage. Lancet 2009;373:1632-44.

22. Keep RF, Hua Y, Xi G. Intracerebral haemorrhage: mechanisms of injury and therapeutic targets. Lancet Neurol 2012;11:720-31.

23. Zheng H, Chen C, Zhang J, et al. Mechanism and Therapy of Brain Edema after Intracerebral Hemorrhage. Cerebrovasc Dis 2016;42:155-69.

24. Hanley DF, Thompson RE, Muschelli J, et al. Safety and efficacy of minimally invasive surgery plus alteplase in intracerebral haemorrhage evacuation (MISTIE): a randomised, controlled, open-label, phase 2 trial. Lancet

Cite this article as: Du Y, Gao Y, Liu HX, Zheng LL, Tan ZJ, Guo H, Wu X, Cui WX, Yang C, Shi YW, Zhou GY, Sun FF, Fan RX, Feng T, Wang P, Wang L, Guo W, Qu Y. Long-term outcome of stereotactic aspiration, endoscopic evacuation, and open craniotomy for the treatment of spontaneous basal ganglia hemorrhage: a propensity score study of 703 cases. Ann Transl Med 2021;9(16):1289. doi: 10.21037/atm-21-1612
Neurol 2016;15:1228-37.

25. Hanley DF, Thompson RE, Rosenblum M, et al. Efficacy and safety of minimally invasive surgery with thrombolysis in intracerebral haemorrhage evacuation (MISTIE III): a randomised, controlled, open-label, blinded endpoint phase 3 trial. Lancet 2019;393:1021-32.

26. Sun G, Li X, Chen X, et al. Comparison of keyhole endoscopy and craniotomy for the treatment of patients with hypertensive cerebral hemorrhage. Medicine (Baltimore) 2019;98:e14123.

27. Kellner CP, Chartrain AG, Nistal DA, et al. The Stereotactic Intracerebral Hemorrhage Underwater Blood Aspiration (SCUBA) technique for minimally invasive endoscopic intracerebral hemorrhage evacuation. J Neurointerv Surg 2018;10:771-6.

28. Kellner CP, Arthur AS, Bain M, et al. MISTIE III: a big step in the right direction. J Neurointerv Surg 2019;11:326-7.

(English Language Editors: K. Brown and J. Chapnick) 


\section{Supplementary}

A
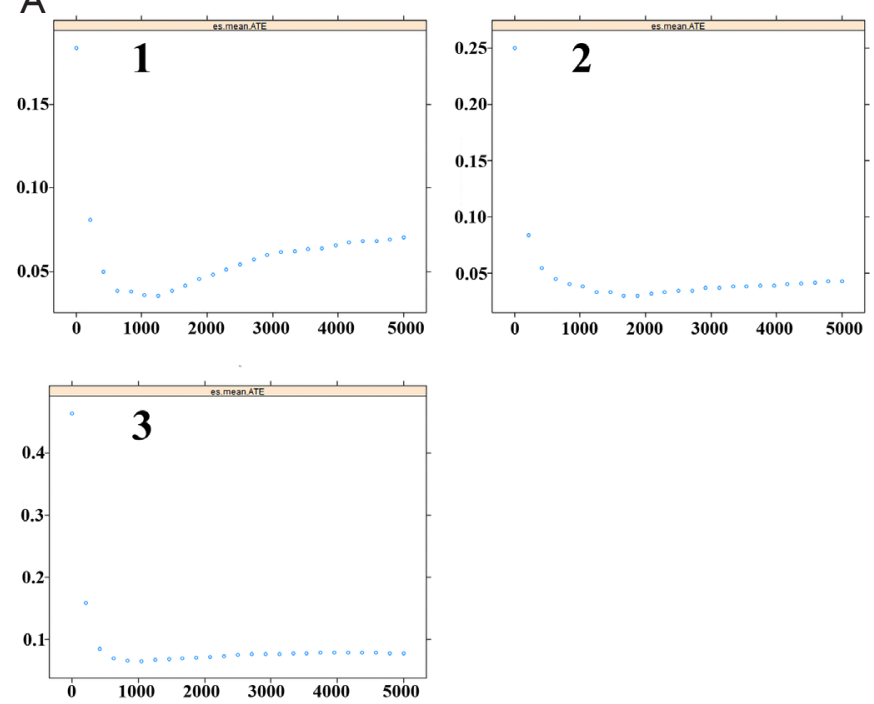

B
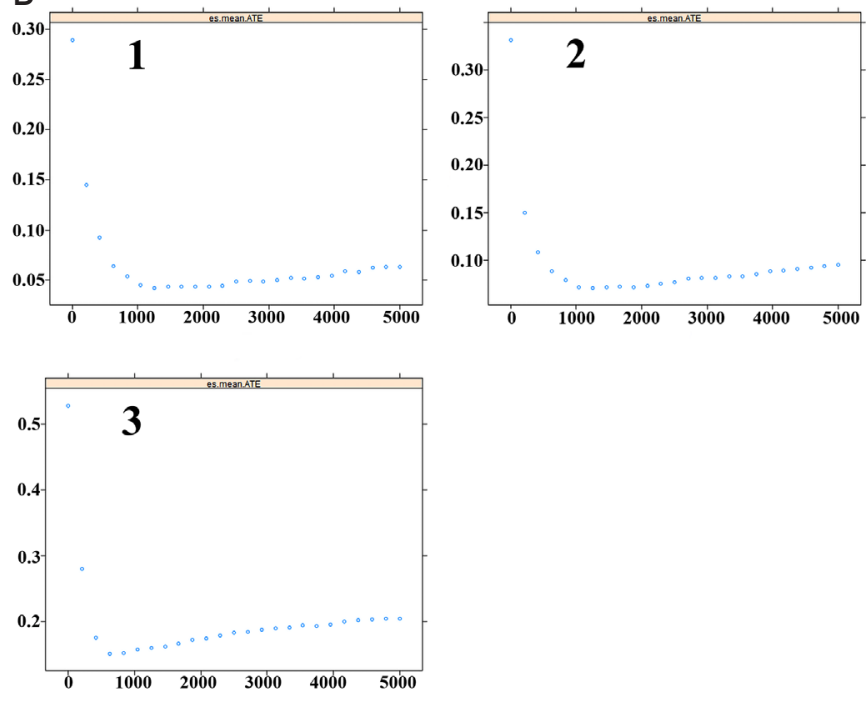

Figure S1 For the entire cohort, generalized boosted model to obtain the optimal balance of the propensity score among groups of the IPTW model associated with mRS score (A) and mortality rates (B). Horizontal axis represents the iteration times and vertical axis represents the balance measure. 1, Balance for EE against others. 2, Balance for SA against others. 3, Balance for OC against others. IPTW, inverse probability of treatment weighted; mRS, modified Rankin Scale; SA, stereotactic aspiration; EE, endoscopic evacuation; OC, open craniotomy.
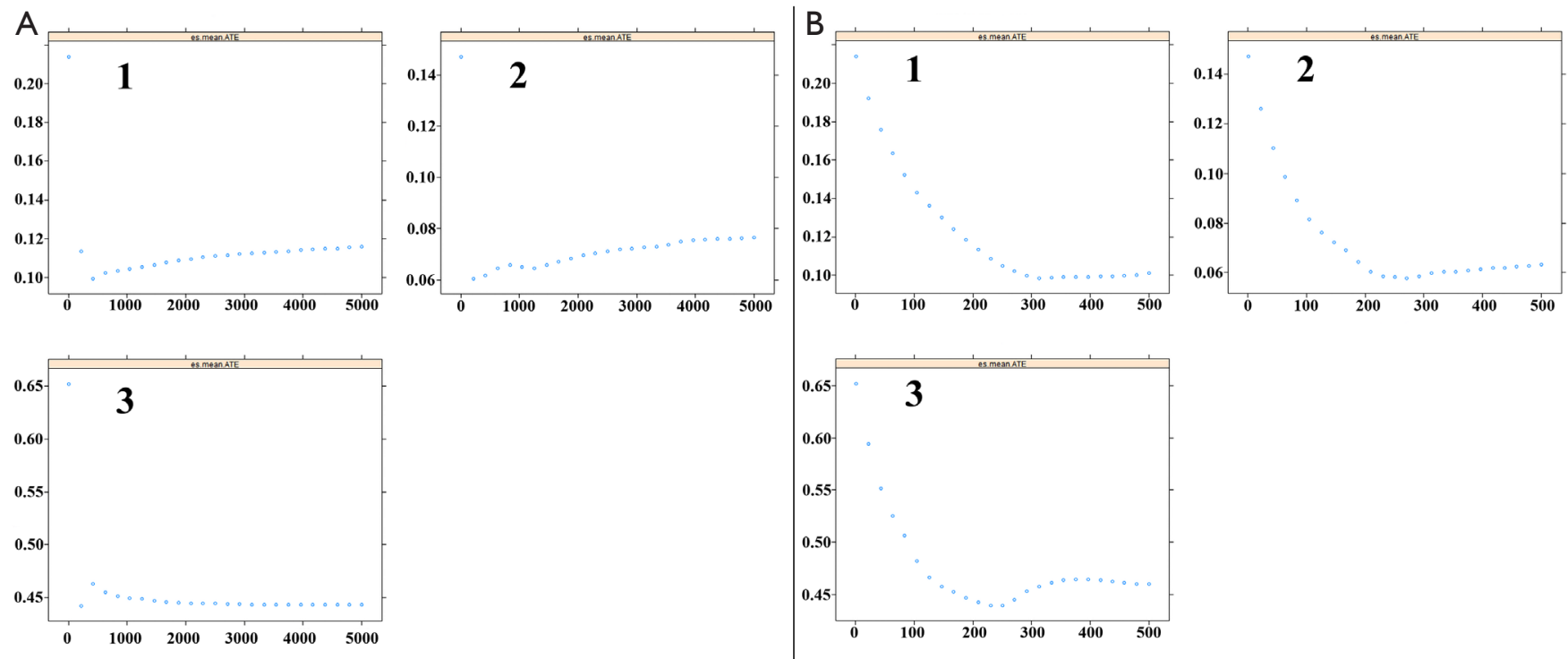

Figure S2 For the subgroup of hematoma volume of 20-40 mL, generalized boosted model to obtain the optimal balance of the propensity score among groups of the IPTW model associated with mRS score (A) and mortality rates (B). Horizontal axis represents the iteration times and vertical axis represents the balance measure. 1, Balance for EE against others. 2, Balance for SA against others. 3, Balance for OC against others. IPTW, inverse probability of treatment weighted; mRS, modified Rankin Scale; SA, stereotactic aspiration; EE, endoscopic evacuation; OC, open craniotomy. 

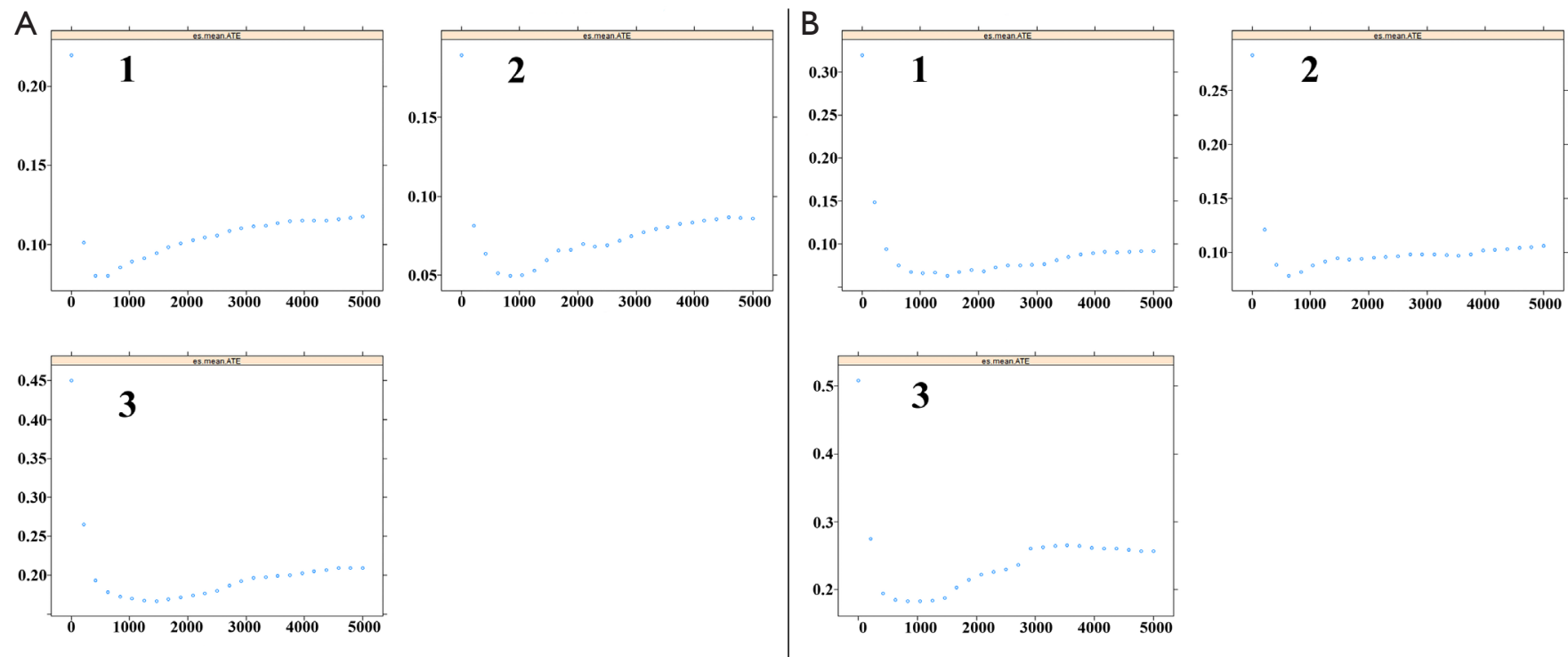

Figure S3 For the subgroup of hematoma volume of 40-80 mL, generalized boosted model to obtain the optimal balance of the propensity score among groups of the IPTW model associated with mRS score (A) and mortality rates (B). Horizontal axis represents the iteration times and vertical axis represents the balance measure. 1, Balance for EE against others. 2, Balance for SA against others. 3, Balance for OC against others. IPTW, inverse probability of treatment weighted; mRS, modified Rankin Scale; SA, stereotactic aspiration; EE, endoscopic evacuation; OC, open craniotomy.

A
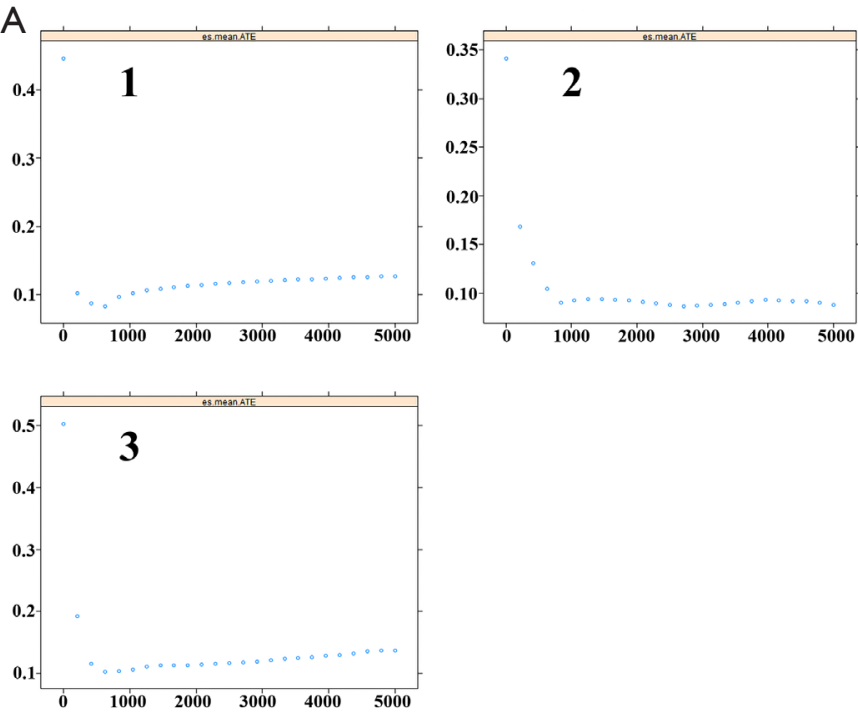
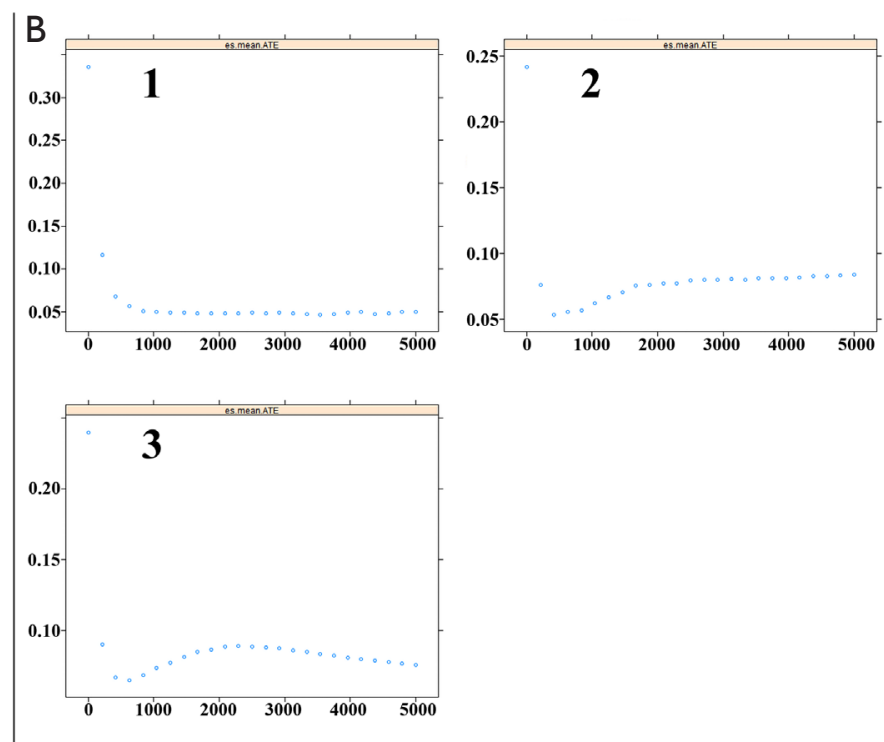

Figure S4 For the subgroup of hematoma volume of $\geq 80 \mathrm{~mL}$, generalized boosted model to obtain the optimal balance of the propensity score among groups of the IPTW model associated with mRS score (A) and mortality rates (B). Horizontal axis represents the iteration times and vertical axis represents the balance measure. 1, Balance for EE against others. 2, Balance for SA against others. 3, Balance for OC against others. IPTW, inverse probability of treatment weighted; mRS, modified Rankin Scale; SA, stereotactic aspiration; EE, endoscopic evacuation; OC, open craniotomy. 

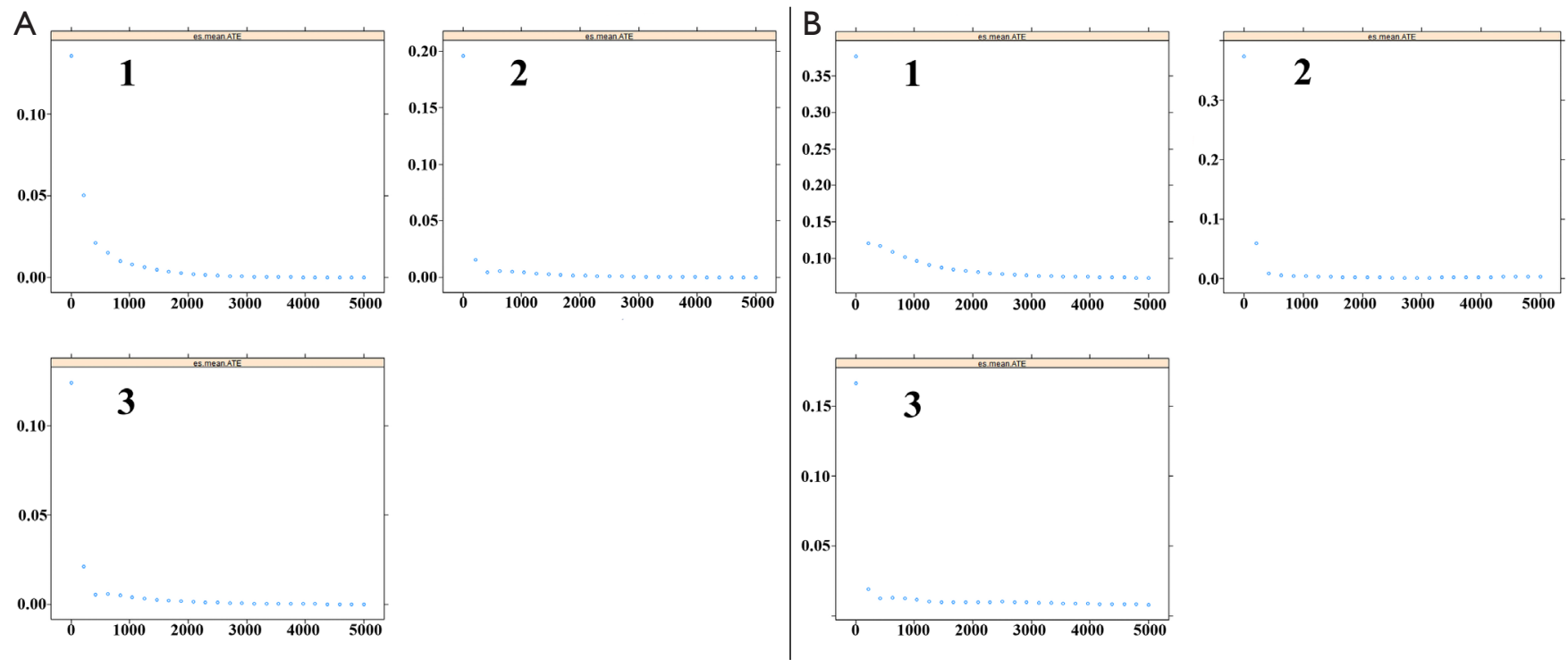

Figure S5 For the subgroup of Glasgow Coma Scale score 3-5, generalized boosted model to obtain the optimal balance of the propensity score among groups of the IPTW model associated with mRS score (A) and mortality rates (B). Horizontal axis represents the iteration times and vertical axis represents the balance measure. 1, Balance for EE against others. 2, Balance for SA against others. 3, Balance for OC against others. IPTW, inverse probability of treatment weighted; mRS, modified Rankin Scale; SA, stereotactic aspiration; EE, endoscopic evacuation; OC, open craniotomy.

A
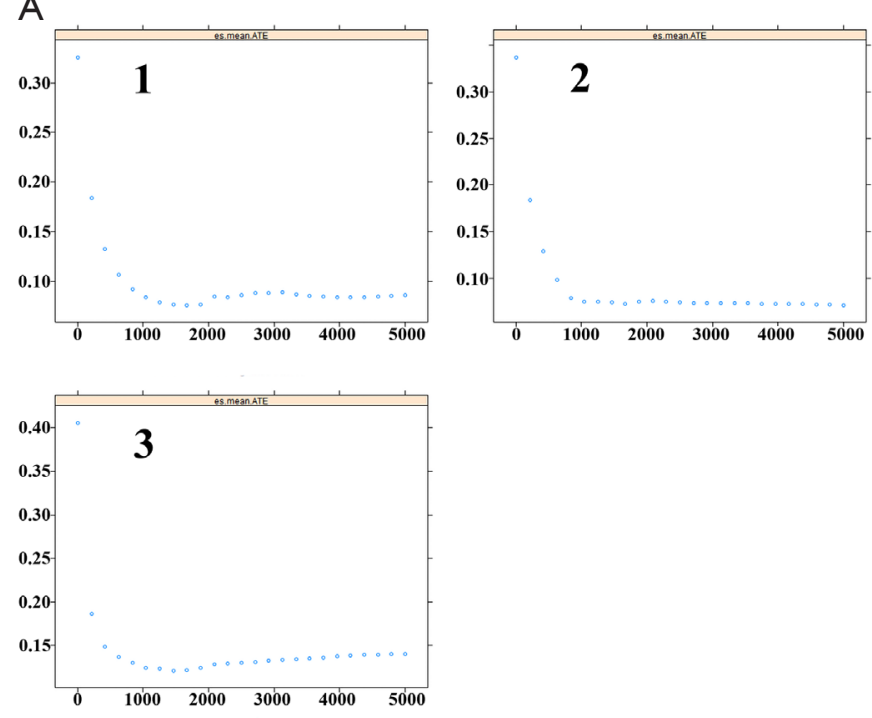

B
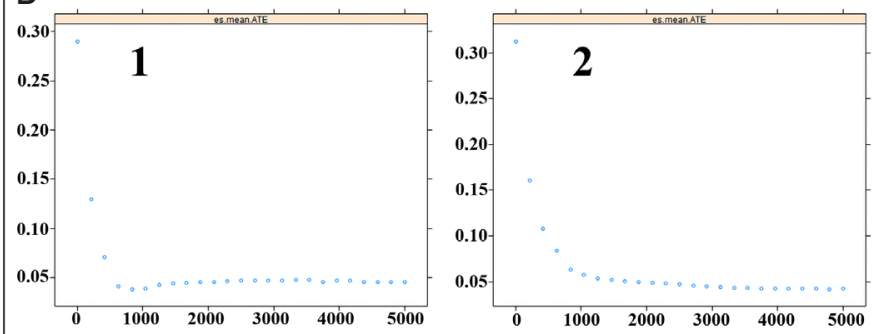

Figure S6 For the subgroup of Glasgow Coma Scale score 6-8, generalized boosted model to obtain the optimal balance of the propensity score among groups of the IPTW model associated with mRS score (A) and mortality rates (B). Horizontal axis represents the iteration times and vertical axis represents the balance measure. 1, Balance for EE against others. 2, Balance for SA against others. 3, Balance for OC against others. IPTW, inverse probability of treatment weighted; mRS, modified Rankin Scale; SA, stereotactic aspiration; EE, endoscopic evacuation; OC, open craniotomy. 

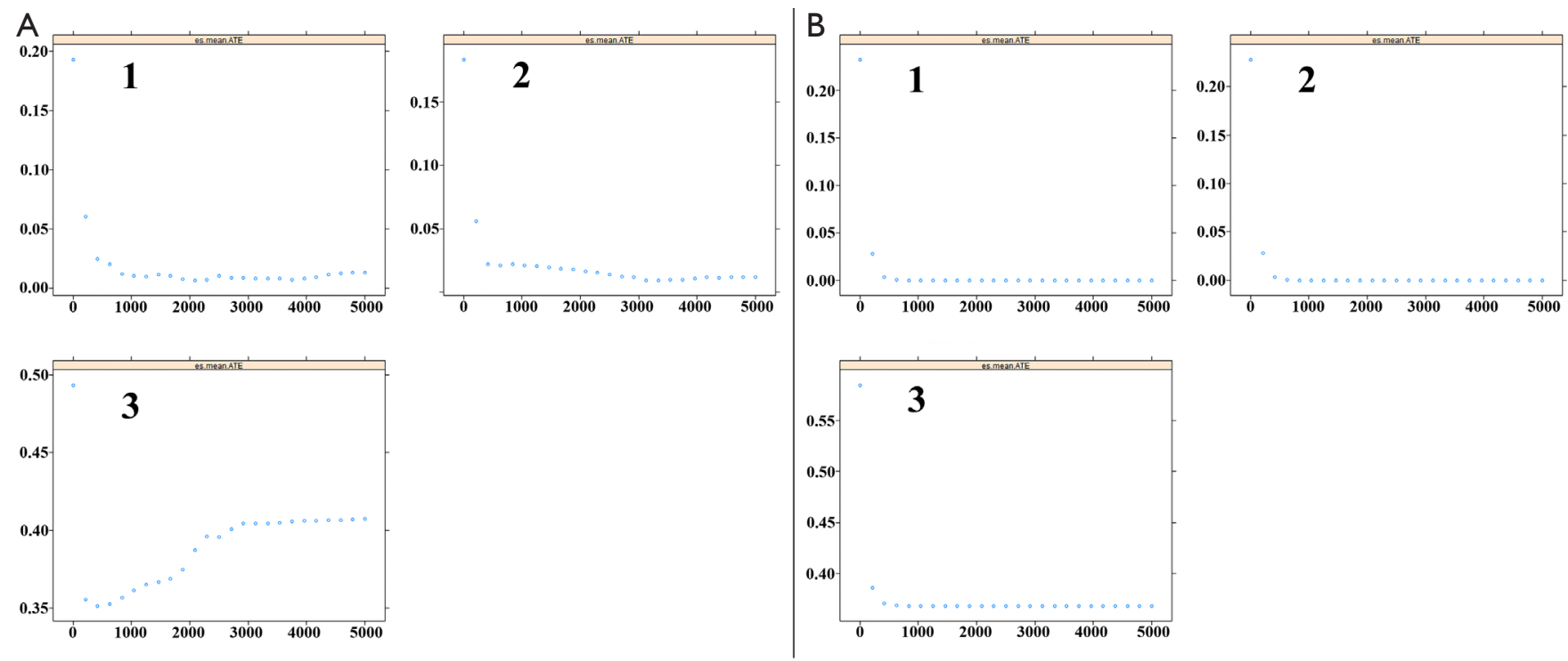

Figure S7 For the subgroup of Glasgow Coma Scale score 9-14, generalized boosted model to obtain the optimal balance of the propensity score among groups of the IPTW model associated with mRS score (A) and mortality rates (B). Horizontal axis represents the iteration times and vertical axis represents the balance measure. 1, Balance for EE against others. 2, Balance for SA against others. 3, Balance for OC against others. IPTW, inverse probability of treatment weighted; mRS, modified Rankin Scale; SA, stereotactic aspiration; EE, endoscopic evacuation; OC, open craniotomy.

A
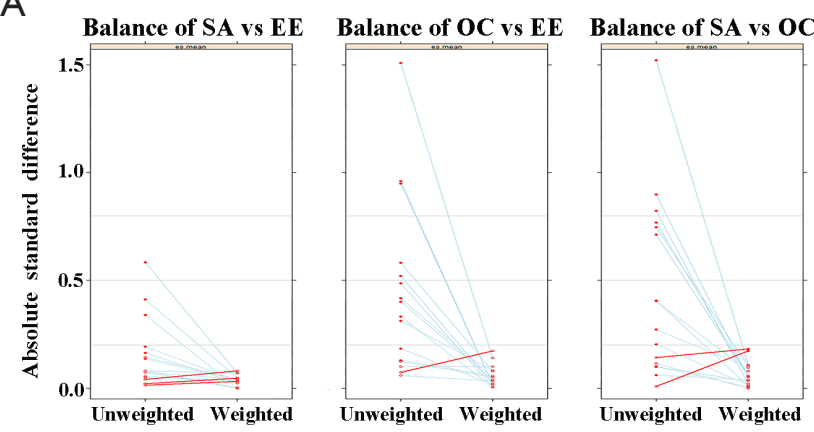

The adjusted covariates: Age, Diabetes, History of craniocerebral disease, GCS score, Herniation,

Hematoma volume, Interval between onset and operation, Decompressive craniectomy, Tracheotomy, Extemal lumbar drainage.
B
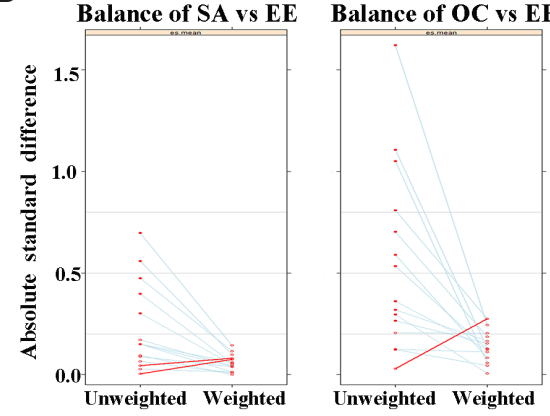

Balance of SA vs OC

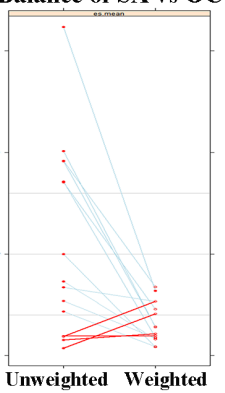

The adjusted covariates: Age, Somking, History of craniocerebral disease, GCS score, Herniation,

Hematoma volume, Interval between onset and operation, Decompressive craniectomy, Lumbar puncture.

Figure S8 For the entire cohort, results of the standardized mean differences to evaluate the effect of weights on the magnitude of each confounding factors of the IPTW model associated with mRS score (A) and mortality rates (B). IPTW, inverse probability of treatment weighted; mRS, modified Rankin Scale; SA, stereotactic aspiration; EE, endoscopic evacuation; OC, open craniotomy; GCS, Glasgow Coma Scale. 
A
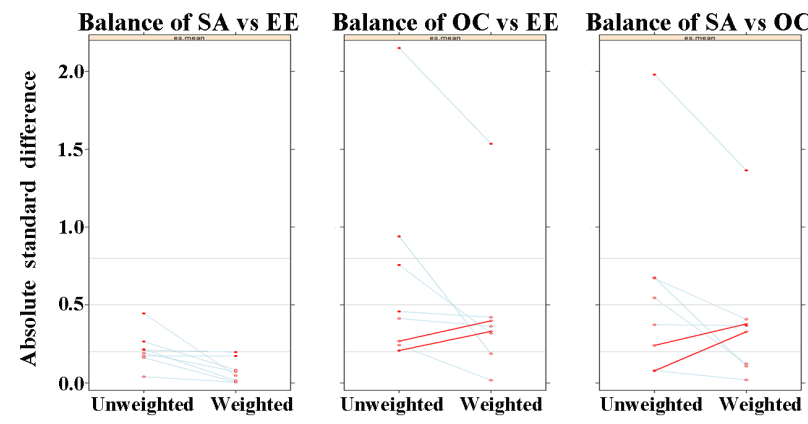

The adjusted covariates: Age, Hypertension, History of craniocerebral disease, GCS score, Herniation,

Decompressive craniectomy.
B
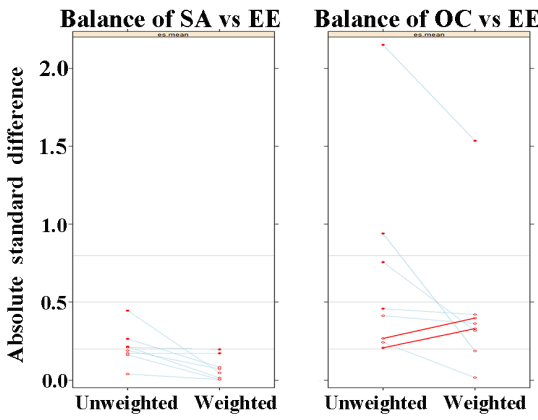

Balance of SA ys OC

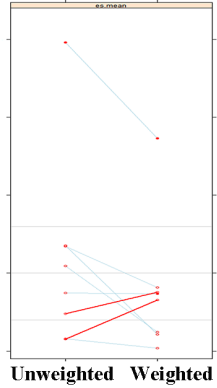

The adjusted covariates: Age, Hypertension, History of craniocerebral disease, GCS score, Herniation, Decompressive craniectomy.

Figure S9 For the subgroup of hematoma volume of 20-40 mL, results of the standardized mean differences to evaluate the effect of weights on the magnitude of each confounding factors of the IPTW model associated with mRS score (A) and mortality rates (B). IPTW, inverse probability of treatment weighted; mRS, modified Rankin Scale; SA, stereotactic aspiration; EE, endoscopic evacuation; OC, open craniotomy; GCS, Glasgow Coma Scale.

A
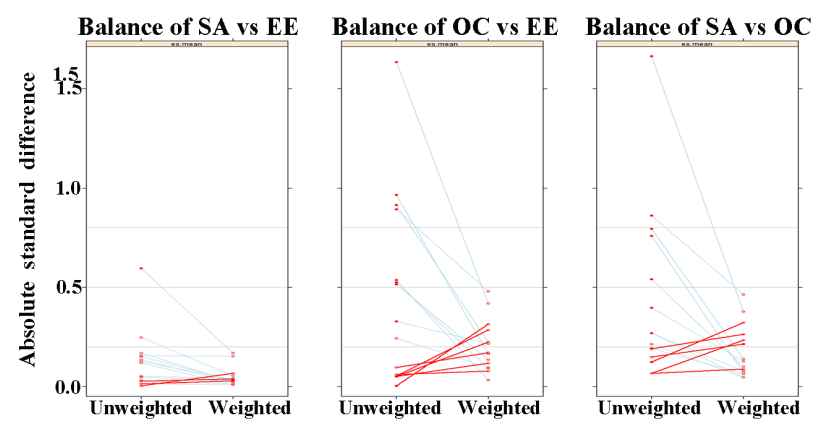

The adjusted covariates: Sex, Age, Somling, Diabetes,

History of craniocerebral disease, GCS score, Herniation,

Interval between onset and operation, Decompressive

craniectomy, Tracheotomy, Extemal lumbar drainage.
B
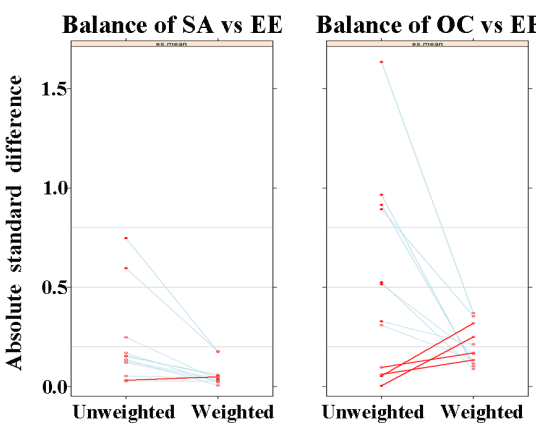

Balance of SA vs OC

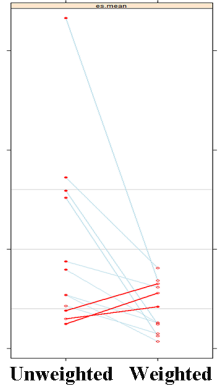

The adjusted covariates: Age, Somling, Diabetes, GCS score, Herniation, Interval between onset and operation, Decompressive craniectomy, Lumbar puncture.

Figure S10 For the subgroup of hematoma volume of $40-80 \mathrm{~mL}$, results of the standardized mean differences to evaluate the effect of weights on the magnitude of each confounding factors of the IPTW model associated with mRS score (A) and mortality rates (B). IPTW, inverse probability of treatment weighted; mRS, modified Rankin Scale; SA, stereotactic aspiration; EE, endoscopic evacuation; OC, open craniotomy; GCS, Glasgow Coma Scale. 
A
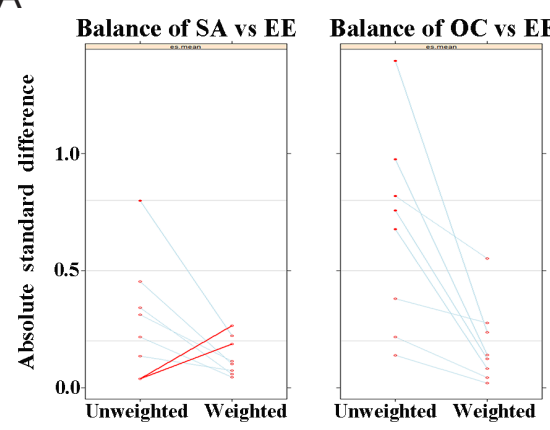

Balance of SA vs OC

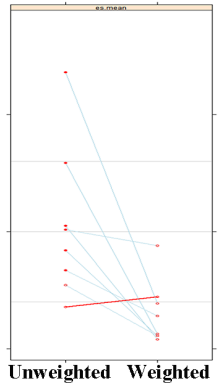

The adjusted covariates: Age, Hypertension, GCS score,

Herniation, Decompressive craniectomy, Lumbar puncture.
B

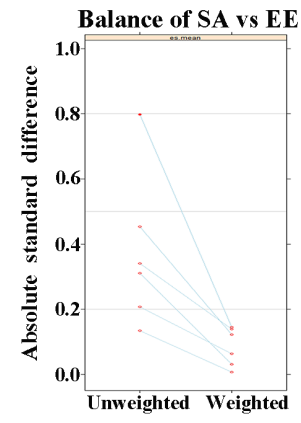

Balance of $\mathrm{OC}$ vs $\mathrm{E}$

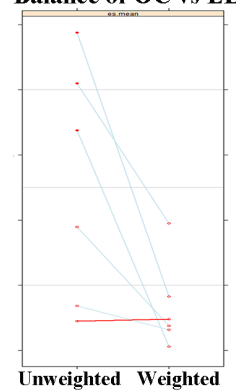

Balance of SA vs OC

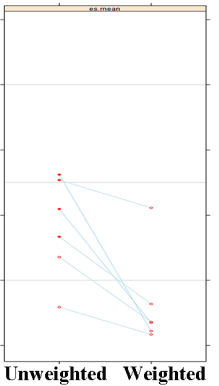

The adjusted covariates: Age, GCS score, Lumbar puncture, Tracheotomy.

Figure S11 For the subgroup of hematoma volume of $\geq 80 \mathrm{~mL}$, results of the standardized mean differences to evaluate the effect of weights on the magnitude of each confounding factors of the IPTW model associated with mRS score (A) and mortality rates (B). IPTW, inverse probability of treatment weighted; mRS, modified Rankin Scale; SA, stereotactic aspiration; EE, endoscopic evacuation; OC, open craniotomy; GCS, Glasgow Coma Scale.

A
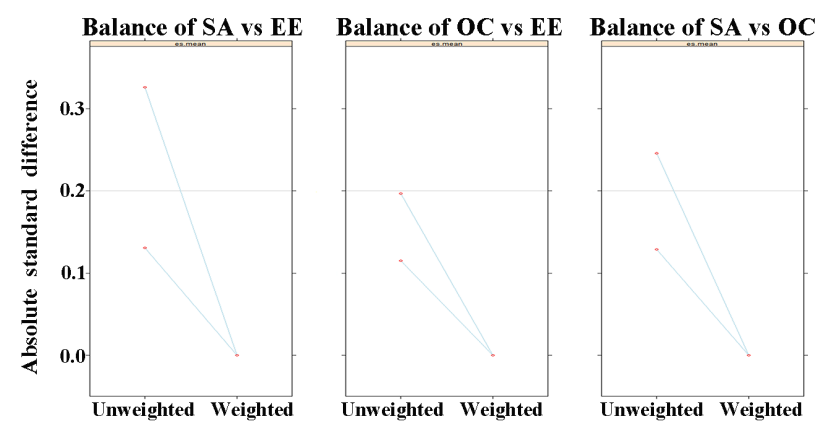

The adjusted covariates: Sex, History of craniocerebral disease.
B
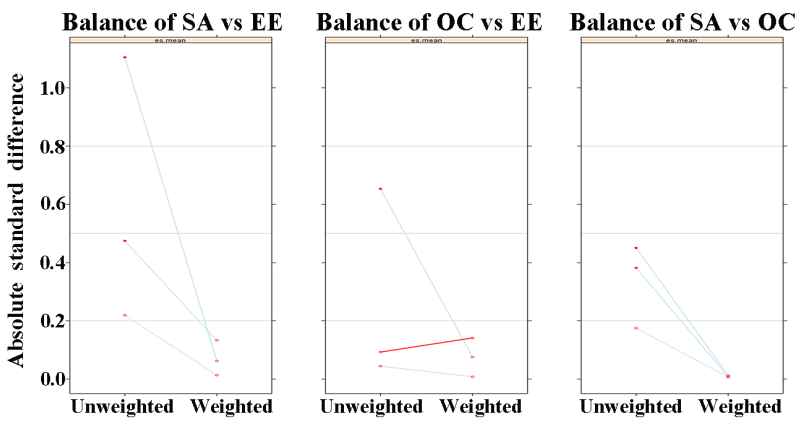

The adjusted covariates: Age, Lumbar puncture,

Tracheotomy.

Figure S12 For the subgroup of Glasgow Coma Scale score 3-5, results of the standardized mean differences to evaluate the effect of weights on the magnitude of each confounding factors of the IPTW model associated with mRS score (A) and mortality rates (B). IPTW, inverse probability of treatment weighted; mRS, modified Rankin Scale; SA, stereotactic aspiration; EE, endoscopic evacuation; OC, open craniotomy. 

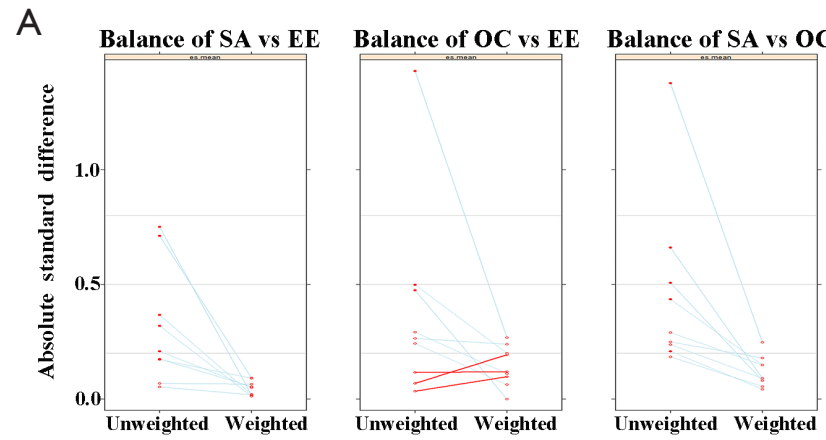

The adjusted covariates: Age, Somling, Diabetes, History of craniocerebral disease, Herniation, Hematoma volume,

Decompressive craniectomy, Lumbar puncture.
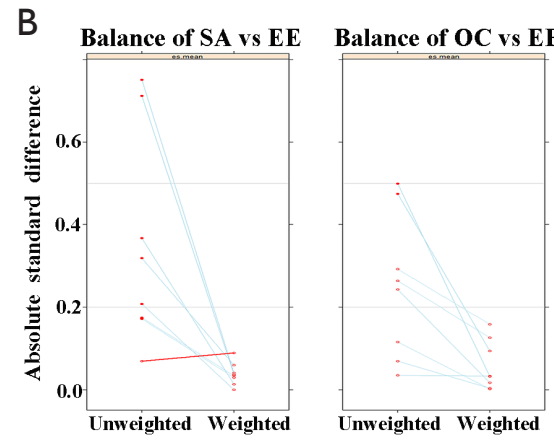

Balance of SA vs OC

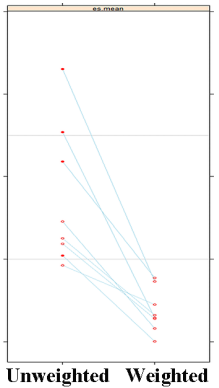

The adjusted covariates: Age, Somling, History of craniocerebral disease, Herniation, Hematoma volume,

Lumbar puncture.

Figure S13 For the subgroup of Glasgow Coma Scale score 6-8, results of the standardized mean differences to evaluate the effect of weights on the magnitude of each confounding factors of the IPTW model associated with mRS score (A) and mortality rates (B). IPTW, inverse probability of treatment weighted; mRS, modified Rankin Scale; SA, stereotactic aspiration; EE, endoscopic evacuation; OC, open craniotomy.

\section{A}
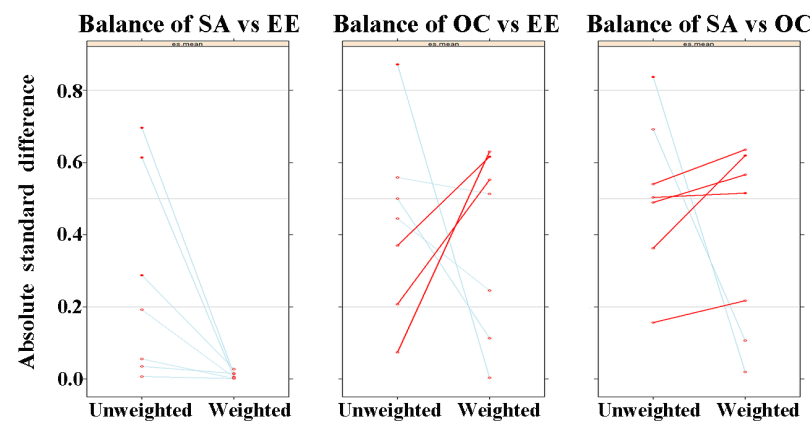

The adjusted covariates: Sex, Age, Hypertension,

Tracheotomy, Hematoma volume.
B
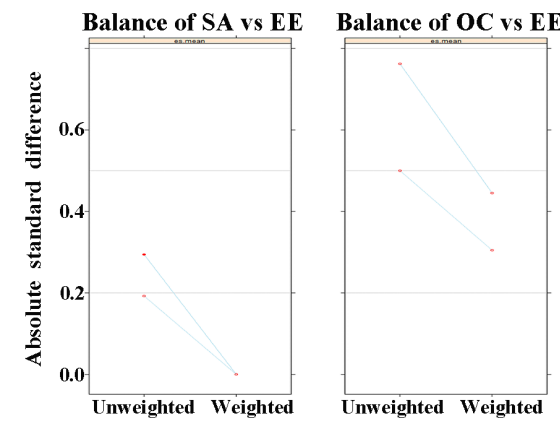

Balance of SA vs OC

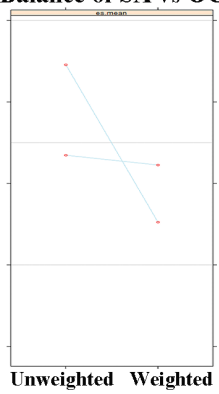

The adjusted covariates: Age, Somling.

Figure S14 For the subgroup of Glasgow Coma Scale score 9-14, results of the standardized mean differences to evaluate the effect of weights on the magnitude of each confounding factors of the IPTW model associated with mRS score (A) and mortality rates (B). IPTW, inverse probability of treatment weighted; mRS, modified Rankin Scale; SA, stereotactic aspiration; EE, endoscopic evacuation; OC, open craniotomy. 
Table S1 The inter-group balance tests of possible confounding factors among different surgical techniques in different hematoma volume subgroups

\begin{tabular}{|c|c|c|c|c|c|c|c|c|c|c|c|c|c|c|c|c|}
\hline \multirow{2}{*}{ Characteristic } & \multirow{2}{*}{ Classification } & \multicolumn{5}{|c|}{ Hematoma volume (20-40 mL) } & \multicolumn{5}{|c|}{ Hematoma volume (40-80 mL) } & \multicolumn{5}{|c|}{ Hematoma volume ( $\geq 80 \mathrm{ml}$ ) } \\
\hline & & Total $(n=195)$ & $S A(n=151)$ & $\operatorname{EE}(n=38)$ & $\mathrm{OC}(\mathrm{n}=6)$ & $P$ value & Total $(n=353)$ & $S A(n=149)$ & $E E(n=134)$ & $\mathrm{OC}(\mathrm{n}=70)$ & $P$ value & Total $(n=155)$ & $\mathrm{SA}(\mathrm{n}=43)$ & $\mathrm{EE}(\mathrm{n}=40)$ & $\mathrm{OC}(\mathrm{n}=72)$ & $P$ value \\
\hline Sex & Male & $112(57.44 \%)$ & $90(59.60 \%)$ & $19(50.00 \%)$ & $3(50.00 \%)$ & 0.5259 & $212(60.06 \%)$ & $91(61.07 \%)$ & $85(63.43 \%)$ & $36(51.43 \%)$ & 0.2377 & $122(78.71 \%)$ & $31(72.09 \%)$ & $31(77.50 \%)$ & $60(83.33 \%)$ & 0.3540 \\
\hline Age (years) & $>60$ & $55(58.21 \%)$ & $45(29.80 \%)$ & $8(21.05 \%)$ & $2(33.33 \%)$ & 0.5412 & $146(41.36 \%)$ & $69(46.31 \%)$ & $52(38.81 \%)$ & 25 (35.71\%) & 0.2485 & 60 (38.71\%) & $20(46.51 \%)$ & $16(40.00 \%)$ & $24(33.33 \%)$ & 0.3663 \\
\hline \multirow[t]{3}{*}{ GCS score } & $3-5$ & $20(10.26 \%)$ & $17(11.26 \%)$ & $1(2.63 \%)$ & $2(33.33 \%)$ & $0.0281^{*}$ & $64(18.13 \%)$ & $21(14.09 \%)$ & $9(6.72 \%)$ & $34(48.57 \%)$ & $<0.0001^{\star}$ & 92 (59.35\%) & $23(53.49 \%)$ & $13(32.50 \%)$ & $56(77.78 \%)$ & $<0.0001^{*}$ \\
\hline & $5-8$ & $53(27.18 \%)$ & $35(23.18 \%)$ & $16(42.11 \%)$ & $2(33.33 \%)$ & & $152(43.06 \%)$ & $59(39.60 \%)$ & $61(45.52 \%)$ & $32(45.71 \%)$ & & $53(34.19 \%)$ & $16(37.21 \%)$ & $21(52.50 \%)$ & $16(22.22 \%)$ & \\
\hline & 9-14 & $122(62.56 \%)$ & $99(65.56 \%)$ & $21(55.26 \%)$ & $2(33.33 \%)$ & & $137(38.81 \%)$ & $69(46.31 \%)$ & $64(47.76 \%)$ & $4(5.71 \%))$ & & $10(6.45 \%)$ & $4(9.30 \%)$ & $6(15.00 \%)$ & $0(0.00 \%)$ & \\
\hline Smoking & Yes & $114(58.56 \%)$ & $93(61.59 \%)$ & $18(47.37 \%)$ & $3(50.00 \%)$ & 0.2578 & $210(59.49 \%)$ & $108(72.48 \%)$ & $60(44.78 \%)$ & $42(60.00 \%)$ & $<0.0001^{\star}$ & $84(54.19 \%)$ & $31(72.09 \%)$ & $17(42.50 \%)$ & $36(50.00 \%)$ & $0.0161^{*}$ \\
\hline Diabetes & Yes & $13(6.67 \%)$ & $12(7.95 \%)$ & $1(2.63 \%)$ & $0(0.00 \%)$ & 0.4024 & $24(6.80 \%)$ & $12(8.05 \%)$ & $9(6.72 \%)$ & $3(4.29 \%)$ & 0.5858 & $11(7.10 \%)$ & $3(6.98 \%)$ & $4(10.00 \%)$ & $4(5.56 \%)$ & 0.6799 \\
\hline Hypertension & Yes & 154 (78.97\%) & $121(80.13 \%)$ & $28(73.68 \%)$ & $5(83.33 \%)$ & 0.6600 & 292 (82.72\%) & 125 (83.89\%) & 112 (83.58\%) & $55(78.57 \%)$ & 0.5898 & 129 (83.23\%) & $37(86.05 \%)$ & $35(87.50 \%)$ & 57 (79.17\%) & 0.4452 \\
\hline $\begin{array}{l}\text { History of } \\
\text { craniocerebral disease }\end{array}$ & Yes & $23(11.79 \%)$ & $18(11.92 \%)$ & $5(13.16 \%)$ & $0(0.00 \%)$ & 0.6465 & $40(11.33 \%)$ & $16(10.74 \%)$ & $15(11.19 \%)$ & $9(12.86 \%)$ & 0.8972 & $26(16.77 \%)$ & $8(18.60 \%)$ & $4(10.00 \%)$ & $14(19.44 \%)$ & 0.4095 \\
\hline Herniation & Yes & $8(4.10) \%$ & $7(4.64 \%)$ & $0(0.00 \%)$ & 1 (16.67\%) & 0.1260 & $51(14.45 \%)$ & $16(10.74 \%)$ & $6(4.48 \%)$ & $29(41.43 \%)$ & $<0.0001^{*}$ & $69(44.52 \%)$ & $11(25.28 \%)$ & $11(27.50 \%)$ & $47(65.28 \%)$ & $<0.0001^{*}$ \\
\hline \multirow{3}{*}{$\begin{array}{l}\text { Interval between onset } \\
\text { and operation (hours) }\end{array}$} & $<12$ hours & $70(35.90 \%)$ & $58(38.41 \%)$ & $10(26.32 \%)$ & $2(33.33 \%)$ & $<0.0001^{\star}$ & 157 (44.48\%) & $64(42.59 \%)$ & 49 (36.57\%) & $44(62.86 \%)$ & $0.0025^{*}$ & $114(73.55 \%)$ & 31 (72.09\%) & $26(65.00 \%)$ & 57 (79.17\%) & 0.4942 \\
\hline & 12-24 hours & $82(42.05 \%)$ & $62(41.06 \%)$ & $19(50.00 \%)$ & 1 (16.67\%) & & 137 (38.81\%) & 55 (36.91\%) & $65(48.51 \%)$ & $17(24.29 \%)$ & & $35(22.58 \%)$ & $11(25.58 \%)$ & $12(30.00 \%)$ & $12(16.67 \%)$ & \\
\hline & $\geq 24$ hours & $43(22.05 \%)$ & 31 (20.53\%) & 9 (23.68\%) & $3(50.00 \%)$ & & 59 (16.71\%) & 30 (20.13\%) & 20 (14.93\%) & $9(12.86 \%)$ & & $6(3.87 \%)$ & $1(2.33 \%)$ & $2(5.00 \%)$ & $3(4.17 \%)$ & \\
\hline \multirow{4}{*}{$\begin{array}{l}\text { Rehabilitation } \\
\text { treatment }\end{array}$} & PRT & $96(49.23 \%)$ & $73(48.34 \%)$ & $18(47.37 \%)$ & $5(83.33 \%)$ & 0.0584 & $146(41.36 \%)$ & 52 (34.90\%) & 67 (50.00\%) & $27(38.57 \%)$ & $0.0002^{*}$ & 42 (27.10\%) & $6(13.95 \%)$ & $16(40.00 \%)$ & 20 (27.78\%) & $0.0003^{*}$ \\
\hline & NPRT & 46 (23.59\%) & 31 (20.53\%) & $15(39.47 \%)$ & $0(0.00 \%)$ & & $86(24.36 \%)$ & 39 (26.17\%) & $34(25.37 \%)$ & $13(18.57 \%)$ & & 20 (12.90\%) & $4(9.30 \%)$ & $9(22.50 \%)$ & $7(9.72 \%)$ & \\
\hline & NRT & $23(11.79 \%)$ & $20(13.25 \%)$ & $3(7.89 \%)$ & $0(0.00 \%)$ & & $27(7.65 \%)$ & $8(5.37 \%)$ & 16 (11.94\%) & $3(4.29 \%)$ & & $19(12.26 \%)$ & $1(2.33 \%)$ & $6(15.00 \%)$ & $12(16.67 \%)$ & \\
\hline & mortality & 30 (15.38\%) & 27 (17.88\%) & $2(5.26 \%)$ & $1(16.67 \%)$ & & 94 (26.63\%) & $50(33.56 \%)$ & 17 (12.67\%) & 27 (38.57\%) & & $74(47.74 \%)$ & $32(74.42 \%)$ & $9(22.50 \%)$ & $33(45.83 \%)$ & \\
\hline $\begin{array}{l}\text { Decompressive } \\
\text { craniectomy }\end{array}$ & Yes & $9(4.62 \%)$ & $6(3.97 \%)$ & $0(0.00 \%)$ & $3(50.00 \%)$ & $<0.0001^{\star}$ & $71(20.11 \%)$ & $7(4.70 \%)$ & $8(5.97 \%)$ & $56(80.00 \%)$ & $<0.0001^{\star}$ & 80 (51.61\%) & $12(27.91 \%)$ & $7(37.50 \%)$ & $61(84.72 \%)$ & $<0.0001^{*}$ \\
\hline Tracheotomy & Yes & 27 (13.94\%) & 21 (13.91\%) & $5(13.16 \%)$ & $1(16.67 \%)$ & 0.9726 & $68(19.26 \%)$ & 22 (14.77\%) & 20 (14.93\%) & $26(37.14 \%)$ & $0.0001^{*}$ & $51(32.90 \%)$ & $12(27.91 \%)$ & $15(37.50 \%)$ & $24(33.33 \%)$ & 0.6456 \\
\hline Lumbar puncture & Yes & 52 (26.67\%) & $32(21.19 \%)$ & $18(47.37 \%)$ & $2(33.33 \%)$ & $0.0046^{*}$ & $111(31.44 \%)$ & $24(16.11 \%)$ & $63(47.01 \%)$ & $24(34.29 \%)$ & $<0.0001^{\star}$ & $44(28.39 \%)$ & $5(11.63 \%)$ & $18(45.00 \%)$ & $21(29.17 \%)$ & $0.0034^{*}$ \\
\hline $\begin{array}{l}\text { External lumbar } \\
\text { drainage }\end{array}$ & Yes & $16(8.21 \%)$ & $12(7.95 \%)$ & $4(10.53 \%)$ & $0(0.00 \%)$ & 0.6632 & 40 (11.33\%) & $16(10.74 \%)$ & $15(11.19 \%)$ & $9(12.86 \%)$ & 0.8972 & 22 (14.19\%) & 7 (16.28\%) & $7(17.50 \%)$ & $8(11.11 \%)$ & 0.5844 \\
\hline Re-operation & Yes & $11(5.64 \%)$ & $9(5.96 \%)$ & $2(5.26 \%)$ & $0(0.00 \%)$ & 0.8196 & $14(3.97 \%)$ & $8(5.37 \%)$ & $3(2.24 \%)$ & $3(4.29 \%)$ & 0.3988 & $10(6.45 \%)$ & $3(6.98 \%)$ & $1(2.50 \%)$ & $6(8.33 \%)$ & 0.4778 \\
\hline
\end{tabular}

*, The difference has statistical significance. SA, Stereotactic Aspiration; EE, Endoscopic Evacuation; OC, Open Craniotomy. GCS, Glasgow Coma Scale. PRT, Professional Rehabilitation Treatment, NPRT, Un-professional Rehabilitation Treatment, NRT, No Rehabilitation Treatment. 
Table S2 The inter-group balance tests of possible confounding factors among different surgical techniques in different GCS score subgroups.

\begin{tabular}{|c|c|c|c|c|c|c|c|c|c|c|c|c|c|c|c|c|}
\hline \multirow{2}{*}{ Characteristic } & \multirow{2}{*}{ Classification } & \multicolumn{5}{|c|}{ GCS 3-5 } & \multicolumn{5}{|c|}{ GCS 6-8 } & \multicolumn{5}{|c|}{ GCS 9-14 } \\
\hline & & Total $(n=176)$ & $\mathrm{SA}(\mathrm{n}=61)$ & $\mathrm{EE}(\mathrm{n}=23)$ & $\mathrm{OC}(\mathrm{n}=92)$ & $P$ value & Total $(\mathrm{n}=258)$ & $S A(n=110)$ & $\operatorname{EE}(n=98)$ & OC $(n=50)$ & $P$ value & Total $(\mathrm{n}=269)$ & $\mathrm{SA}(\mathrm{n}=172)$ & $\mathrm{EE}(\mathrm{n}=91)$ & $\mathrm{OC}(\mathrm{n}=6)$ & $P$ value \\
\hline Sex & Male & 116 (65.91\%) & 36 (59.02\%) & 15 (65.22\%) & 65 (70.65\%) & 0.3302 & 151 (28.53\%) & 62 (56.36\%) & 60 (61.22\%) & 29 (58.00\%) & 0.7743 & 179 (66.54\%) & 114 (66.28\%) & 60 (65.93\%) & 5 (83.33\%) & 0.6770 \\
\hline Age (years) & $>60$ & 62 (35.23\%) & 25 (40.98\%) & 7 (30.43\%) & 30 (32.61\%) & 0.4981 & 98 (37.98\%) & 51 (46.36\%) & 30 (30.61\%) & 17 (34.00\%) & 0.0530 & 101 (37.55\%) & 58 (33.72\%) & 39 (42.86\%) & 4 (66.67\%) & 0.1143 \\
\hline Smoking & Yes & $103(58.52 \%)$ & 43 (70.49\%) & $11(47.83 \%)$ & 49 (53.26\%) & 0.0569 & 154 (59.69\%) & $85(77.27 \%)$ & 42 (42.86\%) & 27 (54.00\%) & $<0.0001^{*}$ & 151 (56.13\%) & $104(60.47 \%)$ & $42(46.15 \%)$ & $5(83.33 \%)$ & $0.0335^{*}$ \\
\hline Diabetes & Yes & 13 (7.39\%) & $7(11.48 \%)$ & $1(4.35 \%)$ & $5(5.43 \%)$ & 0.3145 & $21(8.14 \%)$ & $10(9.09 \%)$ & $9(9.18 \%)$ & $2(4.00 \%)$ & 0.4912 & $14(5.20 \%)$ & $10(5.81 \%)$ & $4(4.40 \%)$ & $0(0.00 \%)$ & 0.7484 \\
\hline Hypertension & Yes & 145 (82.39\%) & 51 (83.61\%) & 20 (86.96\%) & 74 (80.43\%) & 0.7279 & 215 (83.33\%) & 94 (85.45\%) & $84(85.71 \%)$ & 37 (74.00\%) & 0.1428 & 215 (79.93\%) & 138 (80.23\%) & $71(78.02 \%)$ & $6(100.00 \%)$ & 0.4226 \\
\hline $\begin{array}{l}\text { History of } \\
\text { craniocerebral } \\
\text { disease }\end{array}$ & Yes & 30 (17.05\%) & 13 (21.31\%) & $2(8.70 \%)$ & 15 (16.30\%) & 0.3763 & 31 (12.02\%) & 11 (10.00\%) & 12 (12.24\%) & $8(16.00 \%)$ & 0.5548 & 28 (10.41\%) & 18 (10.47\%) & 10 (10.99\%) & $0(0.00 \%)$ & 0.6940 \\
\hline \multirow{3}{*}{$\begin{array}{l}\text { Interval between } \\
\text { onset and operation } \\
\text { (hours) }\end{array}$} & $<12$ hours & 128 (72.73\%) & 43 (70.49\%) & 15 (65.22\%) & 70 (76.09\%) & 0.8329 & 132 (51.16\%) & 56 (50.91\%) & 46 (46.94\%) & 30 (60.00\%) & 0.3070 & 81 (30.11\%) & 54 (31.40\%) & 24 (26.37\%) & $3(50.00 \%)$ & 0.4942 \\
\hline & 12-24 hours & $36(20.45 \%)$ & 14 (22.95\%) & 6 (26.09\%) & 16 (17.39\%) & & 91 (35.27\%) & 38 (34.55\%) & $41(41.84 \%)$ & 12 (24.00\%) & & 127 (47.21\%) & 76 (44.19\%) & 49 (53.85\%) & 2 (33.33\%) & \\
\hline & $\geq 24$ hours & $12(6.82 \%)$ & $4(6.56 \%)$ & $2(8.70 \%)$ & $6(6.52 \%)$ & & 35 (13.57\%) & $16(14.55 \%)$ & $11(11.22 \%)$ & $8(16.00 \%)$ & & $61(22.68 \%)$ & 42 (24.42\%) & $18(19.78 \%)$ & $1(16.67 \%)$ & \\
\hline \multirow{3}{*}{$\begin{array}{l}\text { Hematoma volume } \\
(\mathrm{mL})\end{array}$} & $\geq 20-40$ & 20 (11.36\%) & 17 (27.87\%) & $1(4.35 \%)$ & $2(2.17 \%)$ & $<0.0001^{*}$ & 53 (20.54\%) & 35 (31.82\%) & $16(16.33 \%)$ & $2(4.00 \%)$ & $0.0004^{*}$ & $122(45.35 \%)$ & 99 (57.56\%) & $21(23.08 \%)$ & 2 (33.33\%) & $<0.001^{*}$ \\
\hline & $\geq 40-80$ & 64 (36.36\%) & $21(34.43 \%)$ & $9(39.13 \%)$ & 34 (36.69\%) & & 152 (58.91\%) & 59 (53.64\%) & 61 (62.24\%) & 32 (64.00\%) & & 137 (50.93\%) & 69 (40.12\%) & 64 (70.33\%) & 4 (66.67\%) & \\
\hline & $\geq 80$ & 92 (52.27\%) & $23(37.70 \%)$ & 13 (56.52\%) & 56 (60.87\%) & & 53 (20.54\%) & $16(14.55 \%)$ & $21(21.43 \%)$ & 16 (32.00\%) & & $10(3.72 \%)$ & $4(2.33 \%)$ & 6 (6.59\%) & $0(0.00 \%)$ & \\
\hline \multirow{2}{*}{$\begin{array}{l}\text { Rehabilitation } \\
\text { treatment }\end{array}$} & PRT & 52 (29.55\%) & 15 (24.59\%) & $10(43.48 \%)$ & 27 (29.35\%) & $0.0027^{*}$ & $104(40.31 \%)$ & 36 (32.73\%) & 46 (46.94\%) & 22 (44.00\%) & $0.0014^{*}$ & 128 (47.58\%) & 80 (46.51\%) & $45(49.45 \%)$ & $3(50.00 \%)$ & 0.9372 \\
\hline & NPRT & 17 (9.66\%) & $0(0.00 \%)$ & $5(21.74 \%)$ & 12 (13.04\%) & & $55(21.32 \%)$ & 22 (20.00\%) & $27(27.55 \%)$ & $6(12.00 \%)$ & & $80(29.74 \%)$ & 52 (30.23\%) & 26 (28.57\%) & 2 (33.33\%) & \\
\hline $\begin{array}{l}\text { Decompressive } \\
\text { craniectomy }\end{array}$ & Yes & $107(60.80 \%)$ & $13(21.31 \%)$ & $11(47.83 \%)$ & 83 (90.22\%) & $<0.0001^{*}$ & 44 (17.05\%) & 7 (6.36\%) & $4(4.08 \%)$ & 33 (66.00\%) & $<0.0001^{*}$ & $9(3.35 \%)$ & $5(2.91 \%)$ & $0(0.00 \%)$ & 4 (66.67\%) & $<0.001^{*}$ \\
\hline Tracheotomy & Yes & $59(33.52 \%)$ & $13(21.31 \%)$ & $10(43.48 \%)$ & 36 (39.13\%) & $0.0407^{*}$ & $63(24.42 \%)$ & 27 (24.55\%) & 23 (23.47\%) & 13 (26.00\%) & 0.9434 & 24 (8.92\%) & 15 (8.72\%) & $7(7.69 \%)$ & 2 (33.33\%) & 0.1014 \\
\hline Lumbar puncture & Yes & 52 (29.55\%) & $8(13.11 \%)$ & 14 (60.87\%) & 30 (32.61\%) & $<0.0001^{\star}$ & 86 (33.33\%) & 22 (20.00\%) & 49 (50.00\%) & 15 (30.00\%) & $<0.0001^{*}$ & $69(25.65 \%)$ & 31 (18.02\%) & 36 (39.56\%) & 2 (33.33\%) & $<0.001^{*}$ \\
\hline $\begin{array}{l}\text { External lumbar } \\
\text { drainage }\end{array}$ & Yes & 27 (15.34\%) & $9(14.75 \%)$ & $7(30.43 \%)$ & 11 (11.96\%) & 0.0879 & 34 (13.18\%) & $14(12.73 \%)$ & 14 (14.29\%) & $6(12.00 \%)$ & 0.9115 & $17(6.32 \%)$ & $12(6.98 \%)$ & $5(5.49 \%)$ & $0(0.00 \%)$ & 0.7280 \\
\hline Re-operation & Yes & $16(9.09 \%)$ & $6(9.84 \%)$ & $2(8.70 \%)$ & $8(8.70 \%)$ & 0.9691 & $9(3.49 \%)$ & $5(4.55 \%)$ & $3(3.06 \%)$ & $1(2.00 \%)$ & 0.6883 & $10(3.72 \%)$ & $9(5.23 \%)$ & $1(1.10 \%)$ & $0(0.00 \%)$ & 0.2146 \\
\hline
\end{tabular}

* The difference has statistical significance. SA, Stereotactic Aspiration; EE, Endoscopic Evacuation; OC, Open Craniotomy. GCS, Glasgow Coma Scale. PRT, Professional Rehabilitation Treatment, NPRT, Un-professional Rehabilitation Treatment, NRT, No Rehabilitation Treatment. 
Modified Rankin Scale Score

$\square 0 \square 1 \square 2 \square 3 \square 4 \square 5 \square 6$
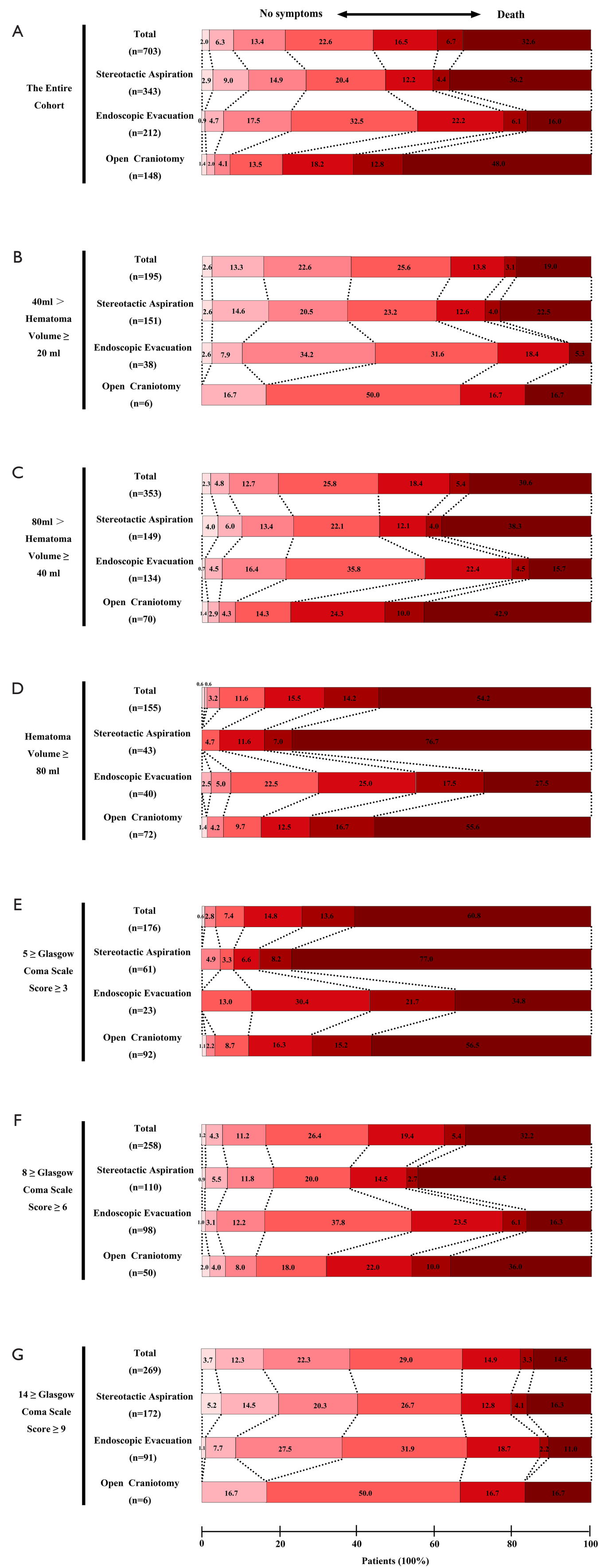

Figure S15 Functional outcomes of patients in each group as assessed by modified Rankin Scale (mRS) score. The scores ranged from 0 to 6 , with 0 indicating no symptoms; 1 , no clinically significant disability; 2 , slight disability; 3 , moderate disability; 4 , moderately severe disability; 5 , severe disability; and 6, death. A score of 4-6 was recognized as a poor outcome. The percentages of patients by the score are shown in each cell. The mRS scores of (A) the entire cohort, (B) patients with a hematoma volume of 20-40 mL, (C) patients with hematoma volume of 40-80 mL, (D) patients with hematoma volume of $\geq 80 \mathrm{~mL}$, (E) patients with GCS score 3-5, (F) patients with GCS score 6-8, (G) patients with GCS score 9-14. GCS, Glasgow Coma Scale. 